\title{
Work With the Beat: How Dynamic Patterns in Team Processes Effect Shared Understanding
}

Philip Cash $^{1 *}$, Elies Dekoninck ${ }^{2}$, Saeema Ahmed-Kristensen ${ }^{3}$

Please cite as: P. Cash, E. Dekoninck, S. Ahmed-Kristensen, "Work With the Beat: How Dynamic Patterns in Team Processes Effect Shared Understanding" Design Studies, IN PRESS, 2020.

DOI: 10.1016/j.destud.2020.04.003

\section{Affiliations}

1 Technical University of Denmark, Denmark

2 University of Bath, UK

3 University of Exeter Business School, UK

*Corresponding author

\section{Highlights}

$>$ A protocol study of team processes and shared understanding development.

$>$ Novel characterisation of dynamic patterns in team processes during design work.

$>$ Identification of taskwork-teamwork interdependency and inter-action heartbeat.

$>$ Identification of cross-level pattern alignment.

$>$ Proposed framework linking process patterns and shared understanding development. 


\begin{abstract}
Shared understanding is central to a design team's performance. While current literature describes general relationships between team interaction and the development of shared understanding, it is not known if or how dynamic patterns in team processes impact this. Using a comparative study, we describe dynamic process patterns that influence the relationship between collaborative design work and shared understanding development. We propose two major patterns that impact shared understanding development: taskwork-teamwork interdependency and inter-action heartbeat, and describe how what we term cross-level pattern alignment moderates their effect. We propose a conceptual model that integrates these insights and provides testable propositions. As such, this work has significant implications for both design researchers and practitioners.
\end{abstract}

Keywords: design activity; design practice; team processes; shared understanding; communication

Shared understanding is key to design team performance (Dong, Kleinsmann, \& Deken, 2013; Stempfle \& Badke-Schaub, 2002) and in particular, the success of distributed development projects (Humayun \& Gang, 2013; Taylor \& Ahmed-Kristensen, 2018). However, shared understanding itself emerges dynamically from intertwined team processes (Grossman, Friedman, \& Kalra, 2017; Marks, Mathieu, \& Zaccaro, 2001). Thus, understanding this dynamic emergence is central to design team performance.

Shared understanding (Ariff, Eris, \& Badke-Schaub, 2013) and team processes (Marks et al., 2001) have received significant research attention. However, Leenders et al. (2016) and Garcia (2005) highlight critical deficits in understanding how dynamic patterns in team processes i.e. fluctuations in activity over time, actually impact performance. Although, a limited number of studies have examined aspects of team processes in design (Dong, 2005; Dong et al., 2013; Shroyer, Lovins, Turns, Cardella, \& Atman, 2018; Stempfle \& Badke-Schaub, 2002), these typically focus on overall relationships between specific actions and shared understanding development, rather than dynamic patterns. For example, Stempfle and Badke-Schaub (2002) limit their treatment of shared understanding development to being a result of question asking, and Dong et al. (2013) describe mental model convergence but do not treat representation activities or interactions between product and process aspects of design work. Further, there is a general bifurcation of the research on team processes across timescales and foci (Cash \& Kreye, 2017; Reiter-Palmon, Sinha, Gevers, Odobez, \& Volpe, 2017). Specifically, the design literature has focused on product related micro-scale cognition and action (Hay, Duffy, et al., 2017), while the management literature has focused on process related macro-scale 
activity (Navarro, Roe, \& Artiles, 2015). Despite this, studies at both the micro and macro scale have highlighted the importance of dynamic patterns in shaping process outcomes (Cash \& Kreye, 2017; Maznevski \& Chudoba, 2000); and limited research has pointed to the importance of dynamic interaction across scales (De Dreu, Nijstad, \& Van Knippenberg, 2008; Gorman, 2014) and product/process foci (Girard \& Robin, 2006). Finally, The lack of theory development in design research is endemic (Cash, 2018), and particularly problematic in areas that are complex and connect research across levels of activity (Bedny \& Harris, 2005), such as in team processes (Leenders et al., 2016; Navarro et al., 2015). Thus, there is a critical need for improved understanding of how dynamic patterns in team processes impact shared understanding development.

In order to address this need, we aim to answer the following research question: What are the relationships between dynamic patterns in team processes and the development of shared understanding? We use protocol analysis on a number of distributed design sessions, which are known to put particular stress on the development of shared understanding (Eris, Martelaro, \& Badke-Schaub, 2014). Throughout we use the basic framing of Activity Theory (Bedny \& Harris, 2005) and Team Processes (Grossman et al., 2017; Marks et al., 2001) to describe design work, summarised in Section 1.2 with key definitions in Figure 1. Thus, we contribute to the understanding of how dynamic patterns in team processes impact shared understanding development.

\section{Theoretical Background}

Shared understanding is an emergent state shaped by team processes (Grossman et al., 2017), and in particular, effective communication (Dong et al., 2013; Eris et al., 2014). Team processes, and subsequent shared understanding, address both taskwork i.e. what is to be done, and teamwork i.e. how the team should interact to achieve this (Crawford \& Lepine, 2013; Mathieu, Heffner, Goodwin, Salas, \& Cannon-Bowers, 2000). Both taskwork and teamwork can take place in a team or alone, they only denote the subjective topic under focus during the described work (Bedny \& Harris, 2005, p. 136; Marks et al., 2001, p. 359). For example, important aspects of shared understanding in design teams include: taskwork related problems (BadkeSchaub, Lauche, Neumann, \& Ahmed, 2007); agreed solutions (Preston, Karahanna, \& Rowe, 2006); critical issue understanding (Ahmed, 2005); teamwork related goal, strategy and vision (Chiu, Hsu, \& Wang, 2006; Espinosa, Slaughter, Kraut, \& Herbsleb, 2007); and role distribution understanding (Badke-Schaub et al., 2007). Generally, shared understanding emerges from human interaction (Ko, Kirsch, \& King, 2005; Pask, 1975), supported by shared context, documentation and work processes (Badke-Schaub et al., 2007; Hinds \& Mortensen, 2005).

The literature on team processes contributes to an overall theoretical understanding of shared work (Grossman et al., 2017; Marks et al., 2001). They refer to the interwoven behavioural, cognitive and social processes linking individuals with one or more common goals (Kozlowski \& Ilgen, 2006). Critical to 
understanding these processes is the multi-level conceptualisation of work provided by Activity Theory (Bedny \& Harris, 2005; Bedny \& Karwowski, 2004).

Activity Theory provides a multi-faceted conceptualisation of work, allowing for the study of interactions between action-level and task-level design team processes dealing with both product and process considerations. This has two main facets. First, activity is defined as a goal-directed system composed of a number of distinct components (Bedny \& Harris, 2005, p. 136):

- Goals: conscious representations of a desired outcome (Bedny \& Harris, 2005). These provide a vector or topic that denotes the direction of activity ${ }^{1}$.

- Tasks: subjectively distinct periods of activity that address high-level goals (Bedny \& Harris, 2005, p. 135; Cash \& Kreye, 2017, p. 4). These deal with the topic focus of the activity e.g. team coordination.

- Actions: behavioural distinct periods of activity that address immediate sub-goals (Bedny \& Harris, 2005, p. 135; Cash \& Kreye, 2017, p. 4). These deal with the basic behaviours used during the activity e.g. knowledge sharing.

Second, activity is multi-level (Bedny \& Harris, 2005, fig. 135):

- Task level: a continuous sequence of tasks addressing various topics in series (Bedny \& Harris, 2005, p. 135; Cash \& Kreye, 2017, p. 4).

- Action level: a continuous sequence of actions reflecting a series of behaviours (Bedny \& Harris, 2005, p. 135; Cash \& Kreye, 2017, p. 4).

Importantly, these two levels happen in parallel. Therefore, at any moment in time, a team process, will be at least described in terms of a task addressing a higher-level topic and an action addressing a sub-goal (Bedny \& Harris, 2005, p. 135; Cash \& Kreye, 2017, p. 4). Further, a single task can be associated with a range of lower level actions. For example, a task, such as goal clarification, could be achieved via a sequence of lower level actions, such as information search, sketching, discussion and so on. As such, both what is done and how it is structured are important and can be separately described at task level and action level in order to understand design team processes. This conceptualisation is illustrated in Figure 1.

\footnotetext{
${ }^{1}$ This follows recent refinements of Activity Theory that disambiguate the 'object' and 'motive' of activity as defined by Bedny and Harris (2005) and discussed by Kaptelinin (2005).
} 


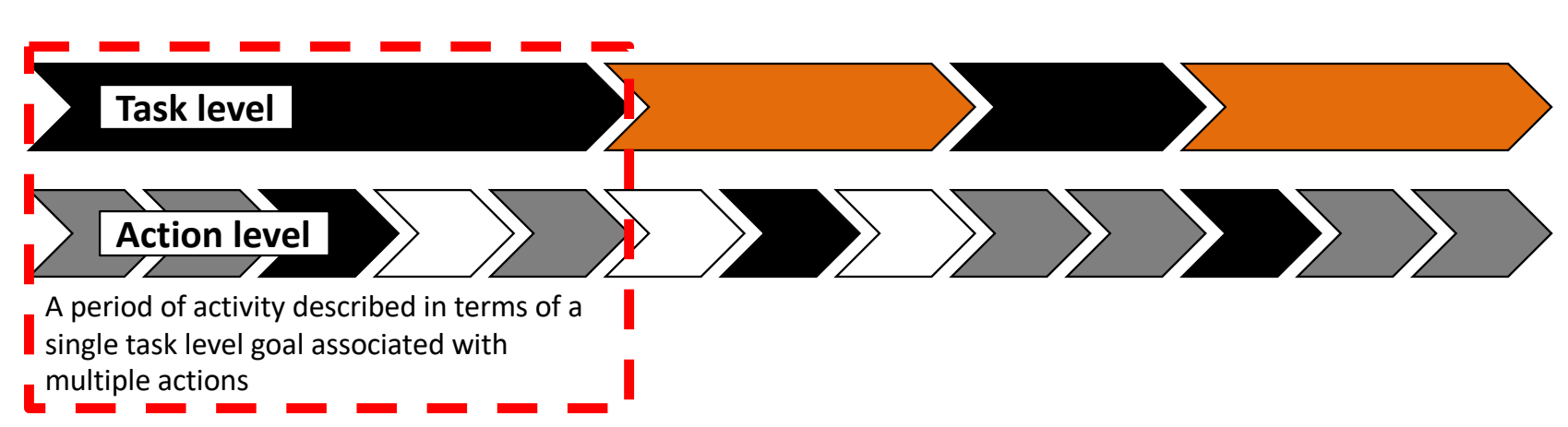

Task level: continuous tasks addressing high-level goals, related to both product focused taskwork: what is to be done, and process focused teamwork: how the team should interact to achieve this, via sequences of actions Action level: continuous sequence of basic behaviours addressing sub-goals

Figure 1: Task level and Action level progression in Activity Theory

While this conceptualisation of design work might appear similar to Schon's theory of reflective practice, it is distinct in a number of important ways. First, Activity Theory conceptualises work processes defined with respect to basic behaviour and cognition (Bedny \& Harris, 2005), while the elements of reflective practice are defined with respect to the design itself e.g. 'framing a problem' or 'moving towards a solution' (Valkenburg \& Dorst, 1998). This makes Activity Theory better suited to synthesising behavioural process research across scales. Second, while tasks provide context for actions - just as frames provide context for moves - Activity Theory connects this to a wider goal framework spanning from overall motivation to cognitive operation (Bedny \& Karwowski, 2004). This allows Activity Theory to conceptualise dynamic variations in behaviour at different levels. Thus, Activity Theory provides a foundation for linking design process discussions across scales as well as to conceptualisations of team processes and shared understanding, which also build on behavioural and cognitive foundations (Grossman et al., 2017).

\subsection{Team Processes and Shared Understanding Development}

Current research shows that while greater knowledge sharing is generally linked to increased shared understanding (Dong et al., 2013; Hult, Ketchen, \& Slater, 2004), Maznevski and Chudoba (2000) suggest that this relationship is influenced by the distribution of knowledge sharing over time. Critically, little is known about how dynamically emerging patterns of interaction between actions and tasks effects shared understanding development. Generally, research has either focused on a single level e.g. Dong et al. (2013), or if considering multiple levels, not treated interactions between processes in terms of driving shared understanding development e.g. Stempfle and Badke-Schaub (2002).

At the action level (Hay, McTeague, et al., 2017; Reiter-Palmon et al., 2017), a number process features have been associated with shared understanding development. Most notably there has been a focus on question asking in the design literature. For example, the positive effect of deliberate question asking and feedback has been demonstrated by Mulder et al. $(2002,2004)$ and Cash et al. (2017). Authors have also described the role of questioning in linking facts, reasoning and context, primarily within an individual (rather than team) 
context (Aurisicchio, Ahmed, \& Wallace, 2007; Aurisicchio, Bracewell, \& Wallace, 2010). This has led to a general characterisation of question asking as critically linked to shared understanding development (Eris, 2002; Qu \& Hansen, 2008; Stempfle \& Badke-Schaub, 2002). Further, authors such as Paletz et al. (2017) and Wiltschnig et al. (2013) have described fluctuations in uncertainty perception and various design behaviours at the sub-goal level (i.e. action level). While such research has led to models at this level, for example by Mulder et al. (2004) or Reimer et al. (2006), these cannot be directly mapped to team processes over the course of a whole session where multiple low-level actions and high-level topics of discussion are intertwined (Navarro et al., 2015). And again, those studies do not account for dynamically emerging patterns of interaction between the two levels.

At the task level (Maznevski \& Chudoba, 2000; Reiter-Palmon et al., 2017), a number of models link team processes and shared understanding development but provide only limited descriptions of dynamics (Leenders et al., 2016). Most notably there has been a focus on static cause-effect relationships in information processing and communication (Hult et al., 2004; Sarin \& O'Connor, 2009). For example, while it is generally held that more communication equates to better team performance (Hult et al., 2004; Pemartín, Rodríguez-Escudero, \& Munuera-Alemán, 2018), a number of authors highlight the effect of process dynamics (Leenders et al., 2016; Sivasubramaniam, Liebowitz, \& Lackman, 2012). While such research has led to the identification of critical variables, for example team coordination (Gilson, Maynard, Jones Young, Vartiainen, \& Hakonen, 2015), these do not account for dynamic phenomena (Leenders et al., 2016; Navarro et al., 2015). Further, due to their high level of granularity they do not investigate potential interactions between task-level topics and actions. However, this literature does hold two insights for this research. First, it provides a common language for team processes (Marks et al., 2001). Second, it offers key conceptualisation regarding process dynamics and structure (Leenders et al., 2016; Navarro et al., 2015).

A small number of authors offer descriptions of design work that bridge these levels. However, these do not generally model the link between dynamic patterns in team processes and shared understanding development. For example, Deken et al. (2012) describe how direct questioning coupled with more discursive exchanges can be intertwined to promote shared understanding development, but do not formalise these interactions. Similarly, while Dong et al. (2013) examine how design teams gradually converge on shared understanding using latent semantics (i.e. using natural language processing to evaluate team sharedness (Dong, 2005; Dong et al., 2013)), this gives insight into overall convergence and is ill suited to explaining the interplay between processes at different levels across varied topics and actions. Specifically, latent semantics are not suitable for studying non-verbal interactions in design, such as representation (Cash \& Kreye, 2017; Dong, 2005).

In summary, while it is generally assumed that dynamic patterns in team processes contribute to performance (Gorman, Amazeen, \& Cooke, 2010; Liu, Chen, \& Tao, 2015), this has not been empirically 
described or theoretically modelled, as highlighted by Navarro et al. (2015), Leenders et al. (2016) and ReiterPalmon et al. (2017).

\subsection{Initial Conceptual Framework}

Given these empirical and theoretical gaps, this research aims to improve understanding of dynamic patterns in team processes and their impact on the development of shared understanding during a design session. This will include the development of theory focusing on the relationships between action level and task level dynamics in team processes.

Starting with the multi-level conceptualisation of task and action illustrated in Figure 1, it is important to define the respective topics and basic behaviours at play. Here, topics and behaviours can be defined independently because the levels progress in parallel. Thus, we sub-divide tasks into 12 topics linked to either taskwork or teamwork, and actions into three basic behaviours (see Table 2 in the methodology), following prior research at each level as described below.

First, we define 12 task-level topics representing important aspects of design work, based on the schema of Deken et al. (2012, p. 209). While a number of alternatives exist (for example, the content-process model of Stempfle and Badke-Schaub (2002), the Merged Ontology of Engineering Design (Ahmed \& Storga, 2009; Storga, Andreasen, \& Marjanovic, 2010) or the ontology of Sim and Duffy (2003)) Deken et al. was selected for its applicability in the study context, its demonstrated robustness, and its relatively recent acceptance in the literature. These topics were then grouped with respect to those dealing with the product to be designed i.e. taskwork, and those dealing with the design process itself i.e. teamwork (based on the seminal discussion of team processes by Marks et al. (2001)). This necessitated the splitting of 'decision making' (Deken et al., 2012, p. 209) into taskwork directed 'product decision making' and teamwork directed 'process decision making'. While this separation of taskwork and teamwork aligns with the general distinction between design content and design process by Stempfle and Badke-Schaub (2002) or between product models and process models by Girard and Robin (2006), it follows the already well established team process terminology.

Second, we separate the action level into three basic behaviours important to design work: information action, knowledge sharing action, and representation action, based on the recent work of Cash and Kreye (2017). This separation was adopted due to its conceptual alignment with the current work - both draw on Activity Theory - as well as its proven robustness and recent publication (Cash \& Kreye, 2018, pp. 59-60). Here, definitions refer to basic behaviours irrespective of topics at the task level.

Based on this we were able to characterise any period of design activity in terms of both its directing topic (task level) and its basic behaviour (action level). For example, a period focused on 'taskwork: solution generation' could progress through a series of actions where the team first quietly sketch individual ideas 
(representation action), articulate each sketch to the team (knowledge sharing action), document the number of parts in each sketch (information action), gesture key movements in each idea (representation action), and discuss misunderstandings (knowledge sharing action), as illustrated in Figure 1.

\section{$2 \quad$ Method}

Given our research question and aim to develop theory (Cash, 2018), a qualitative, multi-case research design was adopted (Handfield \& Melnyk, 1998; Robson \& McCartan, 2011, p. 154). Here, a robust means of developing analytical and theoretical generalisability (Robson \& McCartan, 2011, p. 154) is selecting cases that exemplify theoretical opposites i.e. cases where differences are clear enough to develop the theory of interest (Yin, 2013). Contrasting cases can provide insight into the factors that determine different outcomes (Eisenhardt \& Graebner, 2007). Thus, we have two main methodological criteria: 1) cases are differentiated with respect to the amount of shared understanding development and; 2) cases support examination of team processes at task and action level.

\subsection{Selection and Study Set-up}

As a basis for identifying contrasting cases (Handfield \& Melnyk, 1998, p. 324), data from a controlled study was provided by Cash et al. (2017), and was used in this research for five main reasons: appropriate setting of design task and high-quality recordings, quantitative data to support the selection of contrasting cases, 'no intervention' team processes, and additional questionnaire data available to improve validity; each elaborated below. First, the task was appropriate because it required design teams to carry out design development and design process planning, making it ideal for interrogating interactions between taskwork and teamwork. Three-person teams were given a brief to develop a detailed design plan for the collaborative design, and manufacture of a universal camera mount, which could be attached to a range of remotely controlled aerial vehicles. Each team member was allowed 20 minutes to individually search for relevant information, before the team had 75 minutes to jointly develop the design plan, which required them to settle on at least a preliminary development strategy, using a remote computer interface to communicate. See Figure 2.

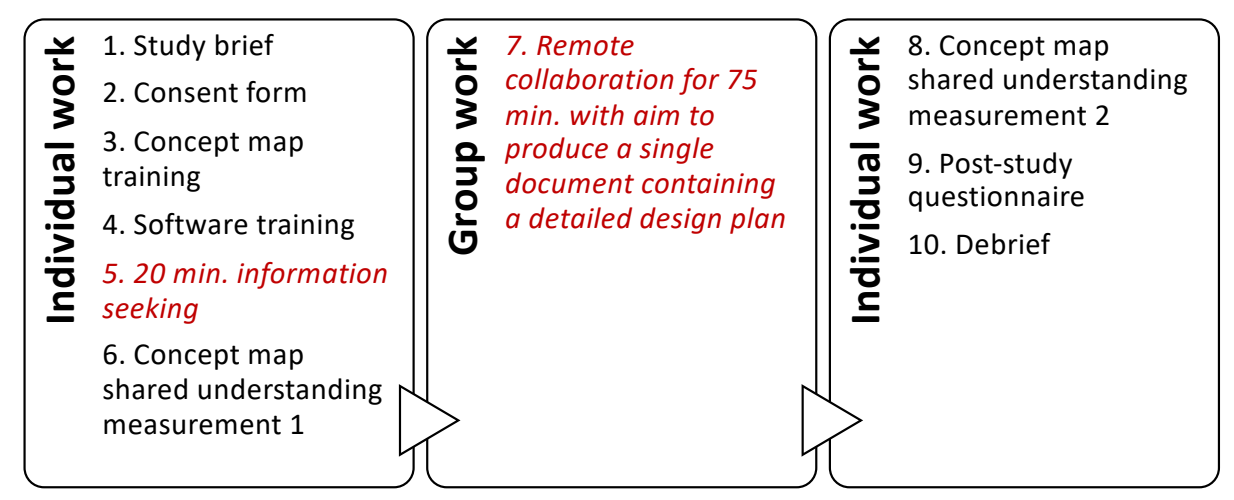

Figure 2: Summary of the study procedure, design work is highlighted in red (steps 5 and 7) 
Second, the study placed design teams in a distributed session. Here, Adobe Connect allowed team members to video talk, type, share information, and sketch in an unconstrained manner (see Section 3.3 for an example of this remote interface in use). This setting is ideal for examining the development of shared understanding in a challenging context (i.e. distributed work) as team processes can be captured in real time. Third, contrasting cases could be readily identified and their differences quantified based on a before/after measure of sharedness, evaluated via concept mapping exercises (6 and 8 in Figure 2) (T. E. Johnson et al., 2007; T. E. Johnson \& O'Connor, 2008). The concept map (step 6 in Figure 2) score of sharedness was based on: the number of concepts with common labels, the number of concepts ordered or connected in common ways, and the number of concepts with common priority indications (Cash et al., 2017, p. 158). Fourth, the control teams from the study of Cash et al. (2017) received no direct intervention targeting their process. They only received an explanation of the communication software used in the experimental setting. This allowed us to select comparable teams based on shared understanding development, and thus create contrasting cases that support isolation of process variables (Shadish, Cook, \& Campbell, 2002). Finally, the extensive nature of the data (summarised in Table 1), with a questionnaire used to gather additional demographic details and perceptions of knowledge sharing (step 9 in Figure 2), supports validity because possible confounding variables could be identified.

Based on this rationale, eight of the 13 control teams used by Cash et al. (2017) were selected. These represented the four teams with the highest positive Shared Understanding (SU) change (delta between concept map measurement 1 and 2, steps 6 and 8 in Figure 2) and the four with the lowest (or even negative) SU change, based on evaluation of a histogram of all teams. For simplicity, we refer to these two groups of teams as 'SU positive' and 'SU negative', reflecting their relative change in shared understanding over the time frame of the study. Table 1 provides details of these two team types. We confirmed this selection using a Mann-Whitney $U$ test to examine increase in shared understanding $(z=2.309 p=0.0209)$ and reduction in number of concepts listed $(z=-1.489 p=0.1489)$; which showed substantial differentiation. We then used the same test to evaluate the measured control variables: pre-session concept map score $(z=-1.169 p=$ $0.2425)$, number of concepts described at session start $(z=0.726 p=0.4678)$, and perception of knowledge sharing quality $(z=-0.289 p=0.7728)$; which revealed no fundamental differences between the teams before the start of the group work. In fact, the SU negative teams had a slightly higher initial shared understanding. Similarly, the two groups were not distinguishable based on the demographic variables, and all but one team contained a mix of female/male participants. As such, confounding explanations for differences in shared understanding development could not be attributed to the team's demographic, perception, or initial level of shared understanding. Thus, further analysis of how the team processes within the session influenced shared understanding development across the contrasting cases is possible. 
Table 1: Identifying contrasting cases: SU positive / negative exemplar teams

\begin{tabular}{|c|c|c|}
\hline & SU positive teams & SU negative teams \\
\hline \multicolumn{3}{|l|}{ Primary selection criteria for SU positive/SU negative examples } \\
\hline $\begin{array}{l}\text { Avg. change in SU based on the difference in pre-post session } \\
\text { concept map score }\end{array}$ & $\begin{array}{c}8 \\
24 \%(S U \text { increase }) \\
\end{array}$ & $\begin{array}{c}-5 \\
-15 \%(S U \text { decrease })\end{array}$ \\
\hline Avg. pre-session concept map score & 11 & 15 \\
\hline \multicolumn{3}{|l|}{ Other variables } \\
\hline Team size & 3 & 3 \\
\hline Avg. age (years) & 27 & 26 \\
\hline Avg. experience (months in industry) & 10 & 9 \\
\hline Avg. total number of concepts described at session start & 46 & 43 \\
\hline $\begin{array}{l}\text { Avg. self-perception of knowledge sharing quality from post } \\
\text { study questionnaire }\end{array}$ & 3.4 & 4.2 \\
\hline
\end{tabular}

\subsection{Analysis of Team Processes}

Given the need to describe process dynamics and interactions (Deeter-Schmelz \& Ramsey, 2003; Navarro et al., 2015) analysis was carried out in three main stages. First, protocol coding was used to elaborate and systematise the team processes data (Gero \& Mc Neill, 1998). Protocol analysis has been highlighted as a key means for investigating team processes (Lehmann-Willenbrock \& Allen, 2018; Movahed-Khah, Ostrosi, \& Garro, 2010). All processes were coded at two levels: 1) task and; 2) action, via the codes in Table 2. Coding used continuous intervals of one second directly in the VCode software. Inter-coder reliability was calculated using Krippendorff's alpha (Krippendorff, 1981) via two raters (an author and an independent third party) on a 300 second data sample where all codes were present. This resulted in an action-level alpha of 0.94, and a task-level alpha of 0.95. Thus, codes are identifiable and mutually exclusive.

Table 2: Protocol codes (after Marks et al. (2001), Deken et al. (2012) and Cash \& Kreye (2017))

\begin{tabular}{|c|c|c|}
\hline Type & Code & Description \\
\hline \multicolumn{3}{|l|}{ Task level } \\
\hline \multirow{7}{*}{$\begin{array}{l}\text { Taskwork topics } \\
\text { (focus on the } \\
\text { product to be } \\
\text { designed) }\end{array}$} & Problem understanding & Analysing the problem, its causes and implications \\
\hline & Requirement finding & Defining adjusting adding or dealing with requirements \\
\hline & Past design analysis & Past solution analysis for the current and other solutions \\
\hline & Design solution explanation & Dealing with potential solutions from prior work \\
\hline & Design solution generation & Developing new product solutions \\
\hline & Design solution analysis & Predicting behaviour and judging solutions \\
\hline & Product decision making & Deciding on the design product \\
\hline \multirow{5}{*}{$\begin{array}{l}\text { Teamwork } \\
\text { topics (focus on } \\
\text { the design } \\
\text { process) }\end{array}$} & Design process & Dealing with the design process for the project \\
\hline & Communication processes & Dealing with meta-communication, context, objectives \\
\hline & Organisational information sharing & Company procedures, information sources, or expertise \\
\hline & Team coordination & Planning current and/or future collaboration \\
\hline & Process decision making & Deciding on the design process \\
\hline \multicolumn{3}{|l|}{ Action level } \\
\hline \multirow{3}{*}{$\begin{array}{l}\text { Actions (basic } \\
\text { behaviours) }\end{array}$} & Information & Dealing with data parts and their manipulation \\
\hline & Knowledge sharing & Dealing with exchange, interpretation and understanding \\
\hline & Representation & Dealing with the manipulation of external representations \\
\hline
\end{tabular}

Second, a number of rounds of qualitative coding were undertaken in order to derive overall themes. I) open coding was used to derive 'first pass' qualitative themes from the raw video and other data (Neuman, 1997, p. 511). II) 'second pass' cross-case analysis was used to refine key themes using axial coding. III) selective 
coding was used to describe exemplars of each theme (Neuman, 1997, pp. 512-514). This resulted in a distilled set of abstract themes. Finally, themes were defined with respect to protocol criteria allowing for a qualitative analysis of all examples/counter-examples as well as quantitative testing of the themes' explanatory power across all SU Positive and SU Negative teams. Throughout, data was examined for explanation of the causal logic underlying the possible theme. The first author carried out the primary analysis in each round, which was then reviewed in a workshop with the whole research team, where the theme wording and explanations were agreed. Table 3 provides a summary of the analysis with an illustration of how the themes developed at each stage.

This process develops themes that draw together insights from multiple data sources, offer consistent explanatory power across cases, and are robust to possible confounding counter examples. Analysis followed best practice for deriving comparative insights from a small sample of in depth cases (Christensen, Johnson, \& Turner, 2011, p. 363; Neuman, 1997, p. 511).

Table 3: Summary of the analysis process

\begin{tabular}{|c|c|c|}
\hline Analysis & Description & Outcomes \\
\hline \multicolumn{3}{|c|}{ First pass quantitative and qualitative data refinement } \\
\hline $\begin{array}{l}\text { Protocol } \\
\text { coding }\end{array}$ & $\begin{array}{l}\text { Coding using the schema } \\
\text { derived from the literature }\end{array}$ & $\begin{array}{l}\text { Structured overview of session timelines in terms of topics and actions } \\
\text { for all sessions enabling the research team to simultaneously examine the } \\
\text { video and protocol timeline }\end{array}$ \\
\hline $\begin{array}{l}\text { Open } \\
\text { coding }\end{array}$ & $\begin{array}{l}\text { Using the videos and } \\
\text { protocol timeline to } \\
\text { examine critical moments, } \\
\text { patterns, and themes }\end{array}$ & $\begin{array}{l}\text { Initial themes 1) integration of product and process discussions, 2) } \\
\text { deliberate structuring of the discussion with representations used at } \\
\text { critical moments, 3) switches between product and process connected to } \\
\text { switches in action with representation use at critical moments }\end{array}$ \\
\hline \multicolumn{3}{|c|}{ Second pass theme identification, elaboration, and refinement } \\
\hline $\begin{array}{l}\text { Axial } \\
\text { coding } \\
\text { round } 1\end{array}$ & $\begin{array}{l}\text { Contrasting first pair of SU } \\
\text { Positive/SU Negative cases } \\
\text { to elaborate the initial } \\
\text { themes }\end{array}$ & $\begin{array}{l}\text { Below we describe the refinements to each initial theme: } \\
\text { 1) Structure used to build up discussion from different perspectives } \\
\text { 2) Structuring of actions, particularly information and representation } \\
\text { 3) Representation and information seeking linked to changes in topic }\end{array}$ \\
\hline $\begin{array}{l}\text { Axial } \\
\text { coding } \\
\text { round } 2\end{array}$ & $\begin{array}{l}\text { Contrasting second pair of } \\
\text { SU Positive/SU Negative } \\
\text { cases to elaborate the } \\
\text { initial themes }\end{array}$ & $\begin{array}{l}\text { 1) Balancing of taskwork and teamwork managed via coordination about } \\
\text { discussion structure } \\
\text { 2) Fewer periods of action used, with a focus on coherent blocks of action } \\
\text { 3) Representation used to link topic and action aspects of the discussion }\end{array}$ \\
\hline $\begin{array}{l}\text { Axial } \\
\text { coding } \\
\text { round } 3\end{array}$ & $\begin{array}{l}\text { Contrasting third pair of } \\
\text { SU Positive/SU Negative } \\
\text { cases to refine the initial } \\
\text { themes }\end{array}$ & $\begin{array}{l}\text { 1) Topic structure built up in conjunction with linked representations of } \\
\text { product, process, and coordination } \\
\text { 2) Actions linked to outcomes and deliberate limitation in the action } \\
\text { 3) Explicit structuring of topic and action planning }\end{array}$ \\
\hline $\begin{array}{l}\text { Axial } \\
\text { coding } \\
\text { round } 4\end{array}$ & $\begin{array}{l}\text { Contrasting final pair of SU } \\
\text { Positive/SU Negative cases } \\
\text { to refine the initial themes }\end{array}$ & $\begin{array}{l}\text { All themes were again observed with few new refinements from prior } \\
\text { observations, suggesting saturation had been achieved. An initial set of } \\
\text { definitions was produced, resulting in the themes seen in Sections 3.1-3.3 }\end{array}$ \\
\hline \multicolumn{3}{|c|}{ Final pass theme illustration and testing } \\
\hline $\begin{array}{l}\text { Selective } \\
\text { coding } \\
\text { round } 1\end{array}$ & $\begin{array}{l}\text { Using all sessions to } \\
\text { identify exemplars for } \\
\text { each theme }\end{array}$ & $\begin{array}{l}\text { Identification of specific examples that represented the archetypal } \\
\text { pattern for each theme. Qualitative examples were developed at this } \\
\text { stage, see for example Figure } 4\end{array}$ \\
\hline $\begin{array}{l}\text { Protocol } \\
\text { selection }\end{array}$ & $\begin{array}{l}\text { Initial theme definitions } \\
\text { linked to the protocol } \\
\text { coding criteria }\end{array}$ & $\begin{array}{l}\text { Systematic identification of all periods related to each theme across the } \\
\text { whole dataset. This also provided theme definitions linked to testable } \\
\text { protocol coding criteria, see for example Section } 3.1\end{array}$ \\
\hline $\begin{array}{l}\text { Selective } \\
\text { coding } \\
\text { round } 2\end{array}$ & $\begin{array}{l}\text { Using all sessions and the } \\
\text { protocol criteria to check }\end{array}$ & $\begin{array}{l}\text { Systematic qualitative evaluation of every theme example and counter } \\
\text { example. Examples were linked to the protocol data such that they could }\end{array}$ \\
\hline
\end{tabular}




\begin{tabular}{|l|l|l|}
\hline & $\begin{array}{l}\text { all examples /counter- } \\
\text { examples }\end{array}$ & $\begin{array}{l}\text { be both qualitative and quantitatively described, see for example Figure } \\
9, \text { where qualitative results are combined with protocol timeline data }\end{array}$ \\
\hline $\begin{array}{l}\text { Statistical } \\
\text { analysis }\end{array}$ & $\begin{array}{l}\text { The final theme } \\
\text { definitions were tested in } \\
\text { the protocol data }\end{array}$ & $\begin{array}{l}\text { Quantitative testing of each theme when comparing all SU Positive } \\
\text { teams' verses SU Negative teams' protocol data. This confirmed the } \\
\text { qualitative analysis, and ensured that each theme provided robust } \\
\text { explanatory power for SU development, see for example Section 3.1 }\end{array}$ \\
\hline
\end{tabular}

3

Results

Three major themes were derived from the analysis, resulting in three main findings as described below. The analysis presents contrasts between SU positive teams and SU negative teams. For each theme we first define it with respect to the protocol data, second, provide statistical analysis to demonstrate its robustness across the sampled teams, and third, illustrate and explain it qualitatively.

\subsection{Taskwork-Teamwork Interdependency}

At the task level, the first theme to emerge was that when taskwork and teamwork are linked in an interdependent pattern this resulted in more effective shared understanding development. Specifically, SU positive teams separate periods of taskwork and teamwork but also deliberately manage switching between them. In order to establish the robustness of these results and concretise the theme we connected these patterns to the protocol analysis. The separation of taskwork and teamwork was characterised as: periods of taskwork and teamwork being separated by at least 60 seconds i.e. there is no overlap between taskwork and teamwork when taking a 60 second rolling average. Overlap is defined as periods where the 60 second rolling average for two or more codes (in this case taskwork and teamwork) are both $>0$. Setting a 60 second overlap is extremely conservative, as the average duration of a topic is circa ten seconds. SU positive teams have less time spent in overlap. Similarly, the deliberate management of switching between taskwork and teamwork was characterised in terms of the protocol analysis as: periods of overlap between taskwork and teamwork corresponding to periods of team coordination. SU positive teams have a higher degree of alignment between periods of overlap and team coordination. These patterns are illustrated in Figure 3. Here, Box 1 highlights a period of overlap coupled with team coordination in the positive example. This is followed by switching between taskwork and teamwork (Box2) and a subsequent period of teamwork with low overlap (Box 3). Contrast this with the negative example, where there are extended periods of overlap without corresponding team coordination (Box 4). Thus, taskwork-teamwork interdependency emerged as a key theme differentiating SU positive from SU negative teams.

Evaluating the separation between periods of taskwork and teamwork across the teams showed significant support for this theme. Specifically, SU positive teams had substantially less overlap between taskwork and teamwork (SU positive mean time in overlap $=1765$ seconds, SU negative mean $=2498$ seconds). Further, while statistical analysis should only be considered as indicative, given the sample size, these results also align with the qualitative findings. Evaluating both Pearson and Spearman correlations, low overlap (i.e. 
lower total time spent in overlap) was significantly linked to percentage decrease in the number of concepts listed by the team (comparing the before/after concept maps (Figure 2)) $r=0.77 p=0.0266$ (rho $=0.79 p=$ 0.0208), and linked to percentage increase in SU, although this second correlation was not significant (Table 1) $r=-0.50 p>0.1$ (rho $=-0.45 p=0.2604$ ). Similarly, examining the deliberate management of switching between taskwork and teamwork by evaluating alignment between periods of overlap and team coordination also showed significant support for this theme. Specifically, we tested the robustness of this proposed interaction via a chi-squared test evaluating instances of overlap with team coordination (i.e. cooccurrence of the two in the same coding interval of one second) verses instances of overlap without team coordination, for both SU positive and SU negative teams, following typical content analysis best practice (Neuendorf, 2017, pp. 359-360). This revealed a significant relationship $\left(X^{2}=11.56 p=0.0007\right)$. Appendix A shows all data used in the correlation analyses. 
Positive Example
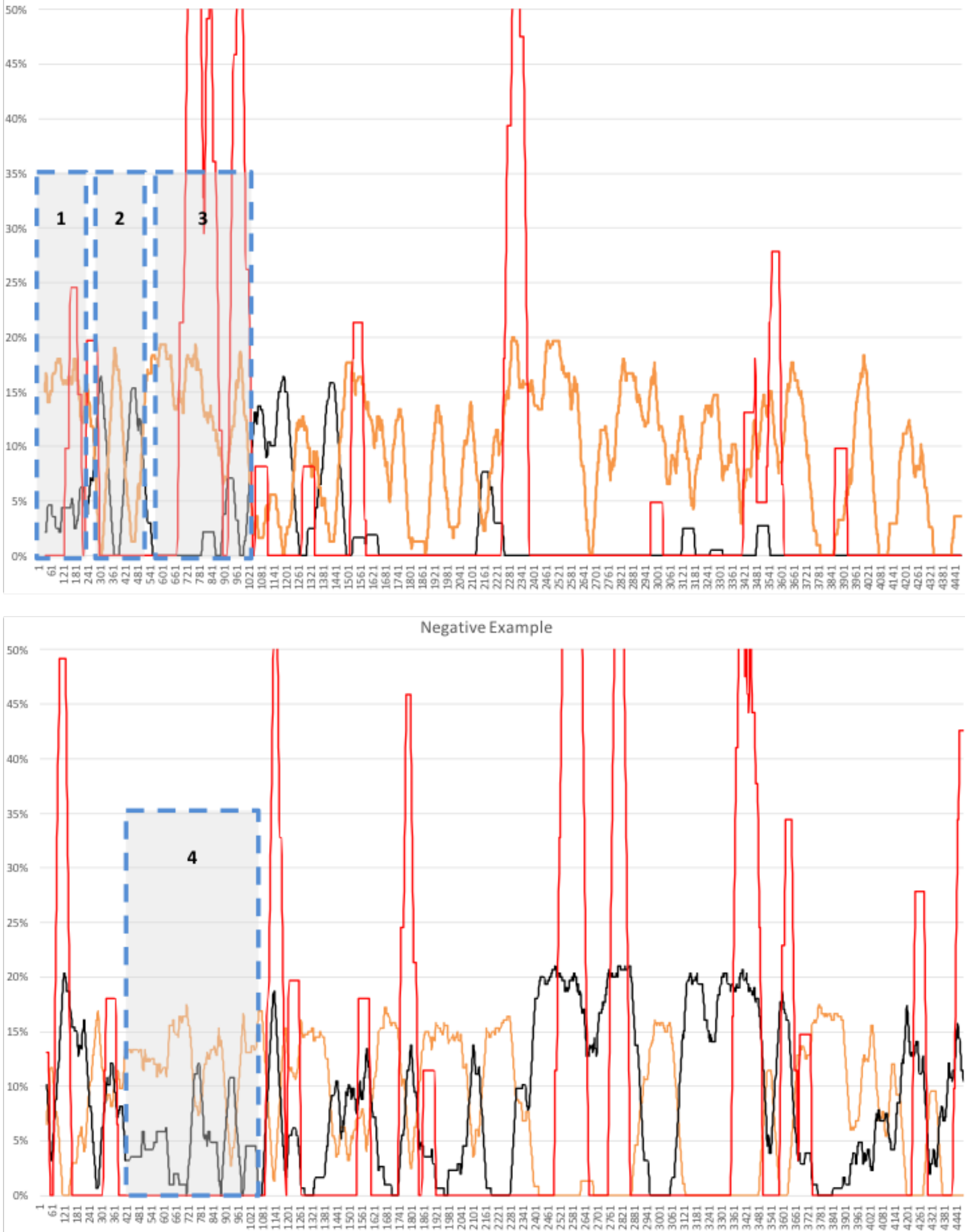

-Taskwork -Teamwork — team coordination

Figure 3: Percentage intensity (y-axis), time (s)(x-axis); Detail of taskwork-teamwork interaction patterns, with team coordination highlighted as a key topic mediating switching in SU positive teams. An example of overlap between taskwork and teamwork is highlighted in Box 4 in the SU negative graph 
Qualitatively, topic periods (i.e. segments where a topic rolling average is $>0$ ) were substantially more defined in SU positive teams, with switches between taskwork and teamwork being deliberately planned and supported by team coordination and representation. Such a switch is illustrated in Figure 4, which shows the transcript of SU positive team 1. Here, the team takes a starting point in taskwork (the specification sketch), to discuss design process considerations (teamwork), switch to taskwork discussion of materials to detail one part of their planning discussion, and then finalise with a teamwork related team coordination sketch. This pattern was repeated, with the team gradually maturing the three related diagrams. These were iterated in parallel throughout the session to deliberately manage the switching between taskwork and teamwork. Specifically, the team would work on the team coordination diagram between periods of work on the problem understanding and design process diagrams (see Figure 4). In this way the team coordination diagram formed the fulcrum around which both taskwork and teamwork diagrams developed.

Finally, taskwork-teamwork interdependency was further supported by the elimination of several possible alternative explanations. First, differences in SU development (Table 1) could not be explained by differences in overall amount of either taskwork or teamwork. Both groups of teams spent similar amounts of time on teamwork: SU positive teamwork mean $=1785$ seconds, SU negative mean = 1947 seconds. The conclusion that neither overall taskwork nor teamwork alone fully explains SU development is supported by correlation analysis, with taskwork and teamwork not being significantly correlated with increase in shared understanding or decrease in number of concepts. Second, overall distribution of taskwork and teamwork on the timeline (beginning to end) were not substantially different across teams i.e. both teams spread their work across the timeline in a similar fashion. A similar finding is evident with respect to the quantity of team coordination: SU positive team coordination mean $=388$ seconds, SU negative mean $=491$ seconds, no significant correlation with SU development or concept reduction. Finally, we again evaluated the robustness of this result across the teams by examining the overall interaction between total taskwork, teamwork and overlap via a chi-squared test, which revealed a significant relationship across SU positive verses SU negative teams $\left(X^{2}=76.28 p=0.0000\right)$.

This theme illustrates how SU positive teams delineate periods of taskwork and teamwork and maintain focus in their discussions. They structure their conversation such that they can deliberately resolve one aspect of the problem/solution at a time, without mixing perspectives and problem elements, particularly between design product and design process. Further, they reflectively manage switches between discussions of taskwork and teamwork in order to maintain alignment between these differing perspectives and ensure continuity and coherence between design product and design process models. 


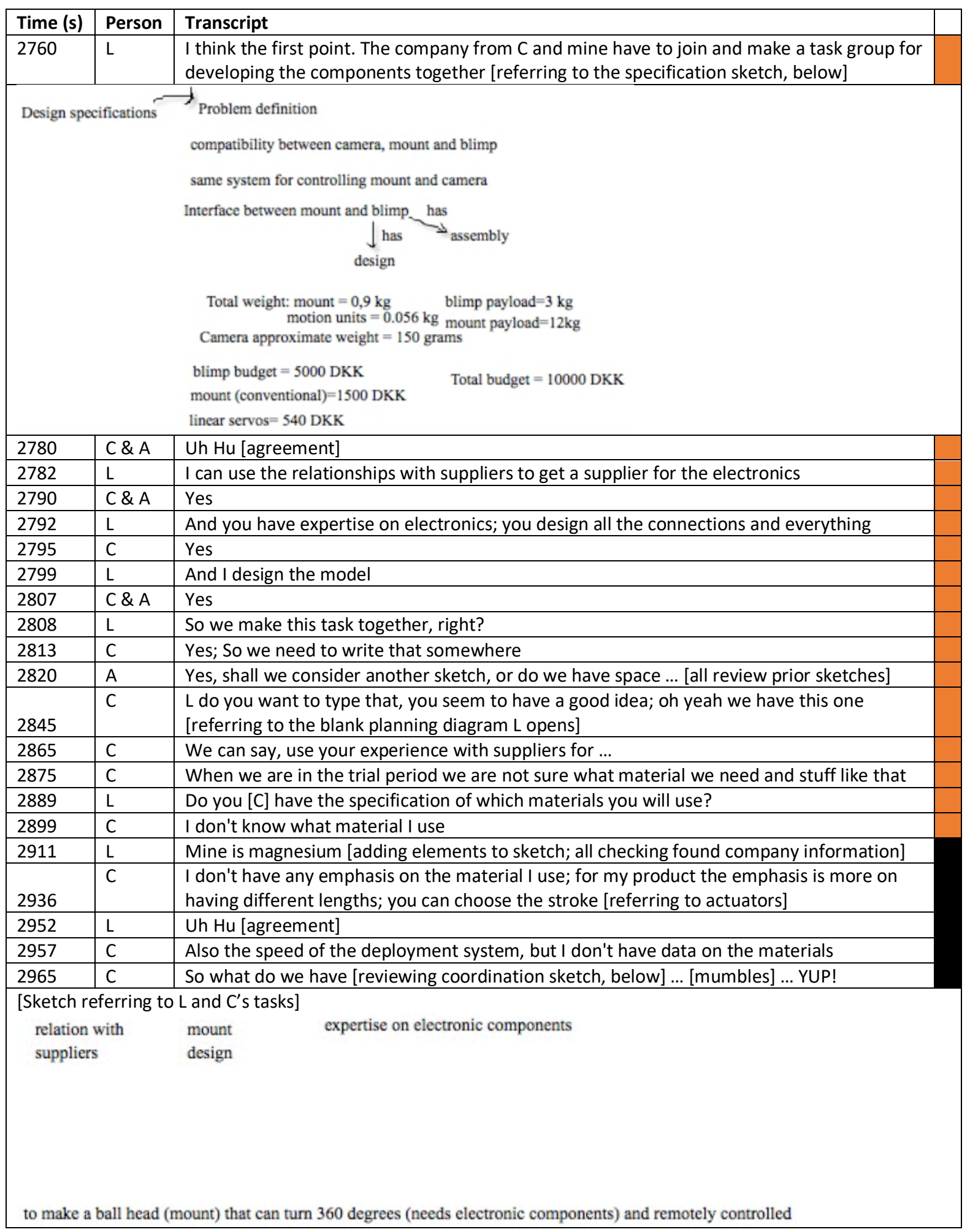

Figure 4: Transcript of a switch between teamwork (denoted in orange) and taskwork (denoted in black) in SU positive team 1 


\section{2}

The second theme to emerge was at the action level. Here, an inter-action 'heartbeat' pattern i.e. regular peaks in action separated by pauses, was linked to effective SU development. Specifically, SU positive teams separated periods of action and followed a periodic action intensity curve. Again, we connected these patterns to the protocol analysis. The separation of actions was characterised as: periods being separated by at least 60 seconds (as with taskwork and teamwork separation). SU positive teams have less time spent in overlap. Similarly, the periodic action intensity curve was identified based on: periodic reduction in level of action below 70\%. SU positive teams have lower intensity. These patterns are illustrated in Figure 5. Contrast the clear, periodic peaks and troughs associated with specific actions in the positive example, where action rhythmically drops below $70 \%$ (For example, note the distinct drop offs in knowledge sharing action highlighted by the arrow annotations in the positive example in Figure 5), and the continuous high intensity action in the negative example, where action is almost constantly over $70 \%$ (Highlighted by the dashed annotation in Figure 5). Appendix B shows the data for all teams.

Evaluating these patterns with the protocol data provided significant support for the theme. For overlap in action SU positive teams had substantially less action overlap (SU positive mean time in overlap $=1777$ seconds, SU negative mean $=2482$ ). Further, low overlap was again correlated with percentage increase in SU, although this was only marginally significant (Table 1) $r=-0.69 p=0.0597$ ( $r$ o $=-0.69 p=0.0580$ ). Examining the overall interaction between total action and action in overlap via a chi-squared test revealed a robust, significant relationship across SU positive verses SU negative teams $\left(X^{2}=32.40 p=0.0000\right)$. Intensity of action was also found to be robust across teams. Correlation analysis showed that as overall intensity of action increased (the sum of the three coded actions) performance decreased in terms of both decrease in concepts $(r=0.91 p=0.0015$; rho $=0.93 p=0.0009)$ and, marginally, increase in SU $(r=-0.69 p=0.0607$; rho $=-0.52 p=0.1827)$.

Qualitatively, action periods were substantially more defined in SU positive teams, with information deliberately translated into knowledge that could be shared by the team. Such a switch is illustrated in Figure 6 , which shows the transcript of SU positive team 3. Here, the team takes a starting point in representation and knowledge sharing regarding the sub-systems and tasks to be considered. Following this they list the sub-systems they are responsible for (information action), which they capture in their planning diagram. They then interpret this information and share thoughts about how to proceed (knowledge sharing). In this way the team structured their discussion throughout the session, gathering the information requiring dissemination and interpretation (e.g. the sub-systems), and then discussing the full set of relevant information as a whole (e.g. listing all sub-systems before coming to discussion of their development). Throughout, they use pauses and down-time to record information and insights via representation. 
Finally, inter-action 'heartbeat' is further supported by the elimination of possible alternative explanations. Specifically, no single action type could be linked to SU development in isolation, rather it was the interaction heartbeat pattern that facilitated the development process. This is supported by correlation analysis, where no individual action type significantly correlates with SU development. Perhaps surprisingly, this also suggests that more knowledge sharing cannot alone be assumed to produce more SU development.

This theme illustrates how SU positive teams delineate periods of action, particularly between knowledge sharing and information action. Different types of action require different cognitive process and have distinctly different foci and behaviour. As such, delineation of action allows the team to more effectively focus their cognition/behaviour, by for example, dealing with a whole chunk of information in one period. SU positive teams support this focusing via representation, which can be used to capture lists of information (allowing a longer period of information action) that can subsequently be discussed and re-structured as a whole (allowing longer and more coherent periods of knowledge sharing action). Finally, SU positive teams use pauses and breaks in conversation to develop these representations, reflect on their prior discussion, and clearly separate periods of action. In contrast, SU negative teams' discussions are continuous, reducing their ability to stop and structure information and knowledge in representations, and further meaning that they continuously mix information and knowledge sharing, rarely completing a list of all relevant information before starting to interpret and restructure this. As such, SU negative teams rarely attain a cohesive overview of all information or relevant knowledge under discussion. 


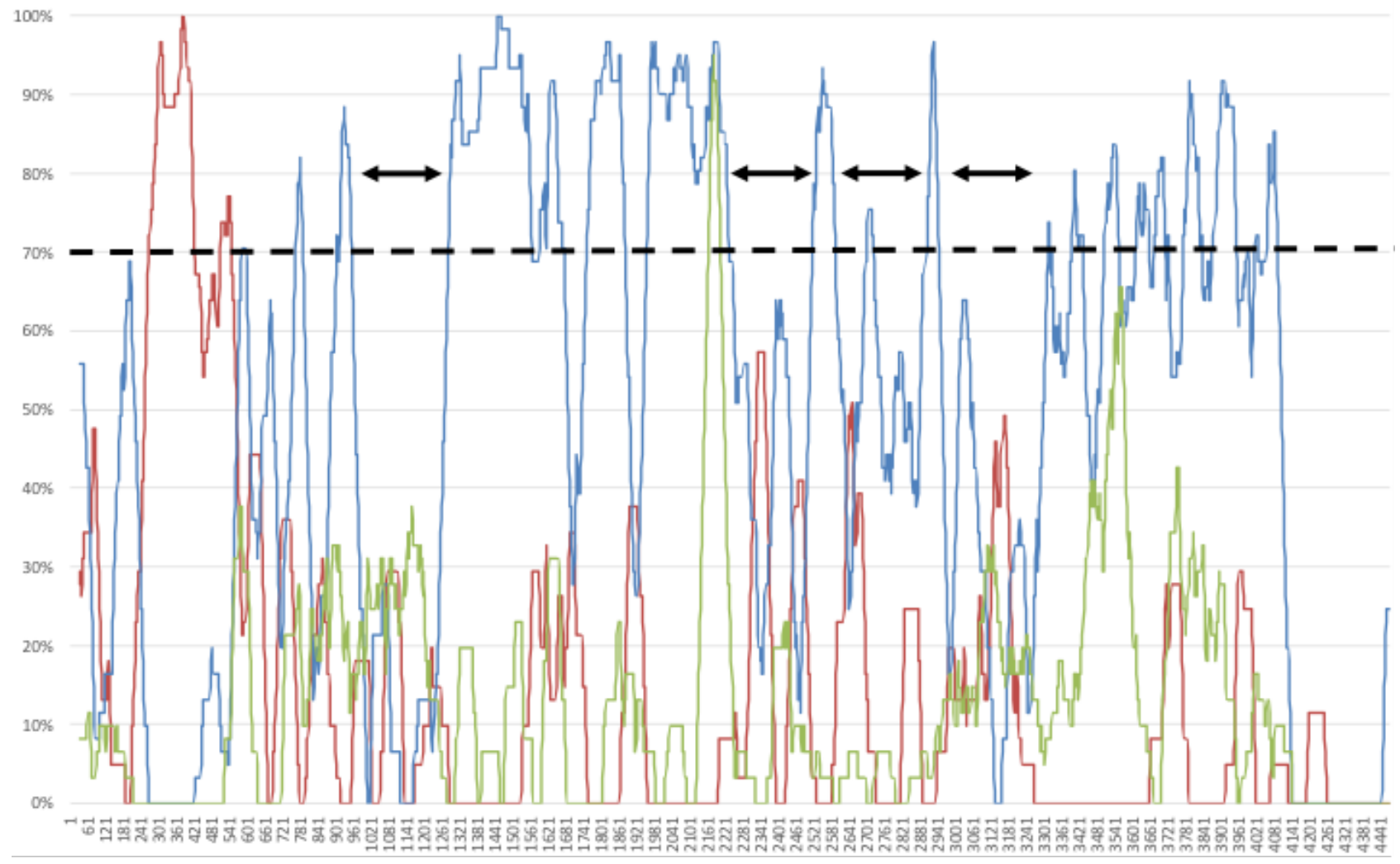

Negative Example

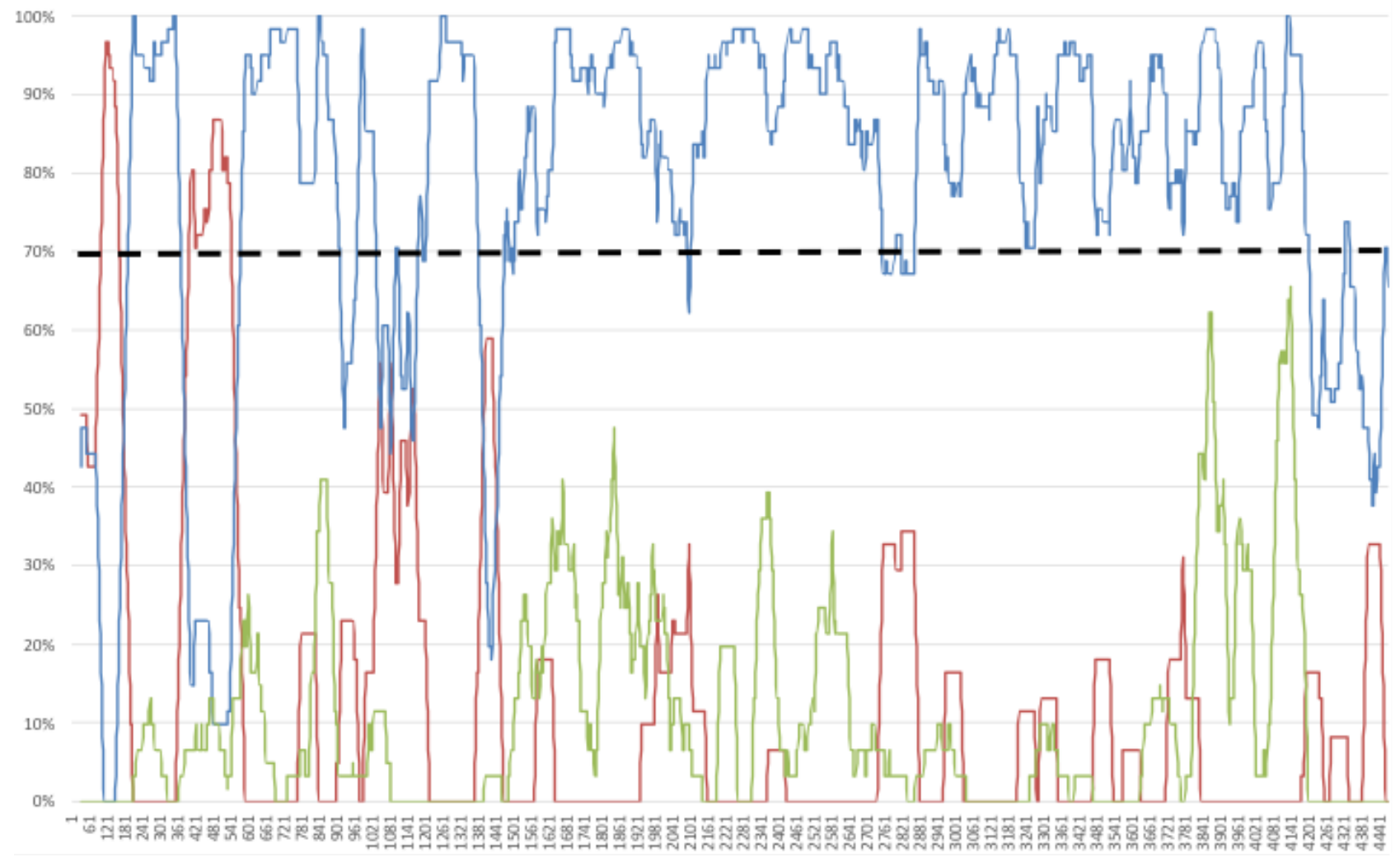

-Information action -Knowledge sharing action - Representation action

Figure 5: Percentage intensity ( $y$-axis), time (s)(x-axis); Action progression with positive: periodic peaks associated with specific actions. Pauses in the action are highlighted by arrows in the positive example 


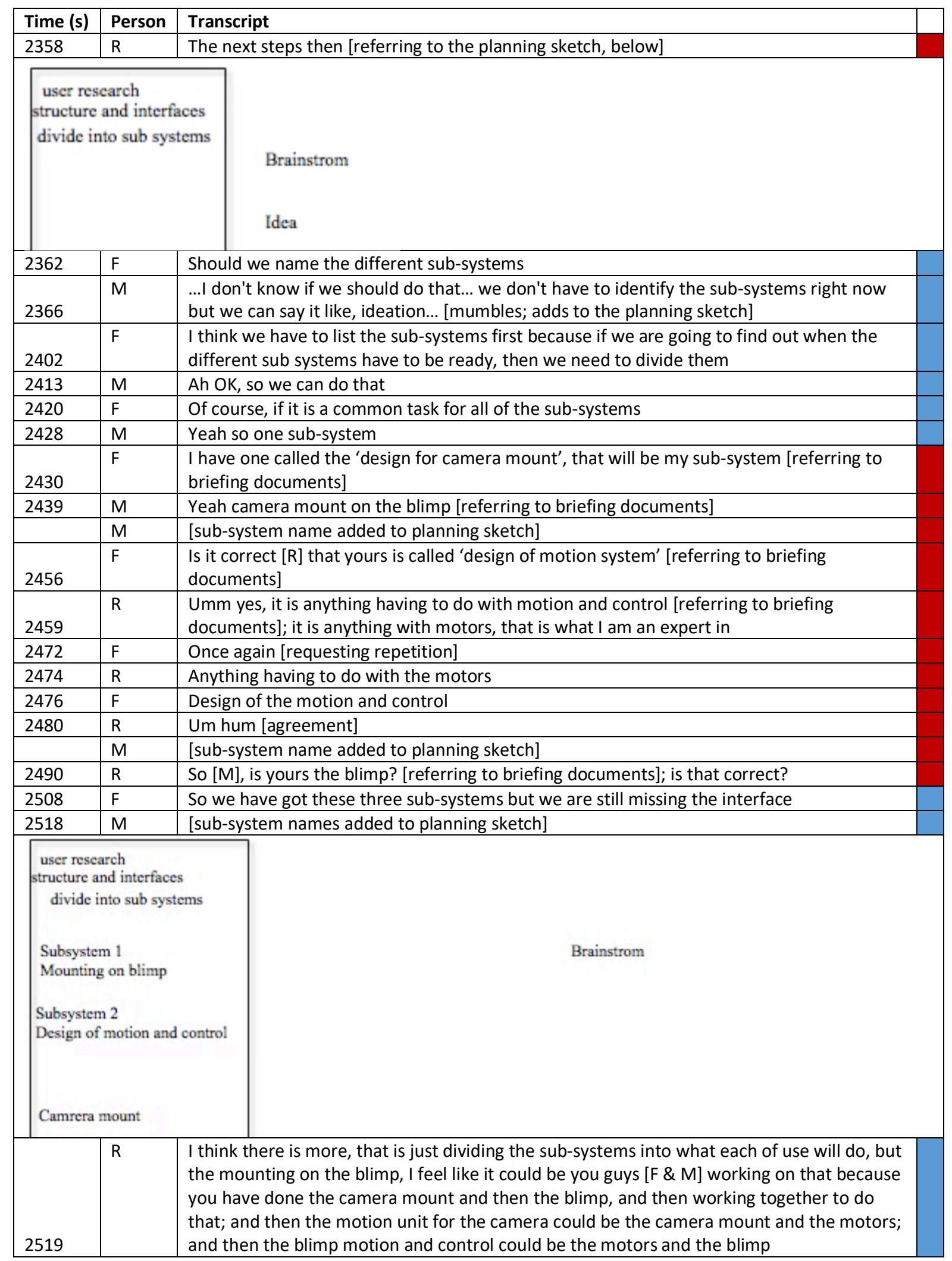

Figure 6: Transcript of a switch between knowledge sharing action (denoted in blue) and information action (denoted in red) in SU positive team 2 


\subsection{Cross-Level Pattern Alignment}

The final theme to emerge was that the impact of the identified process patterns (Sections 3.1 and 3.2) was moderated by cross-level alignment. Further, this alignment was supported by representation-linked team coordination. Specifically, periods of taskwork or teamwork were associated with a single major action coupled with the deliberate linking of team coordination and representation. As before, we connected these patterns to the protocol analysis. Alignment between taskwork/teamwork and action was characterised as: periods where the total action was the same as taskwork or teamwork i.e. there was no 'gap' between the two curves. SU positive teams have less gap. The deliberate linking of team coordination and representation was characterised as: periods of team coordination corresponding to periods of representation. SU positive teams have a higher degree of alignment. The first pattern is illustrated by the positive example in Figure 7 , where there is very close alignment between overall fluctuations in action (i.e. the rolling average of the three action codes, Table 2) and taskwork/teamwork (i.e. the rolling averages of the relevant topic codes, Table 2), highlighted by the arrow annotations. Here, taskwork and teamwork have been displayed with respect to a secondary axis (-20\%) in order to better illustrate alignment. In SU negative teams, there was little evidence of deliberate cross-level alignment. This is illustrated by the negative example in Figure 7 , where there is substantial separation between the overall action curve and the taskwork or teamwork curves, as highlighted by the arrow annotations. Appendix $\mathrm{C}$ shows the data for all teams.

The pattern of intensity alignment between team coordination and representation is illustrated in Figure 8 . In addition to direct alignment, a number of examples were also observed where peaks in team coordination were bracketed by peaks in qualitatively related representation action. In SU negative teams, team coordination was almost never directly linked to peaks in representation action, and typically, representational bracketing was not linked to the team coordination discussion either (based on qualitative evaluation of each occurrence).

Evaluating these patterns with the protocol data provided significant support for the theme across the teams. Specifically, as the degree of alignment between taskwork/teamwork and action decreased (i.e. the 'gap' between the blue line and the orange/black lines in Figure 7) performance increased in terms of both percentage decrease in concepts $(r=0.75 p=0.0331$; rho $=0.76 p=0.0280)$ and increase in SU $(r=-0.65 p=$ 0.0840 ; rho $=-0.40 p=0.3199$ ), although the later was not significant. Evaluating the interaction between total gap and total action via chi-squared showed a significant relationship across SU positive verses SU negative teams $\left(X^{2}=7.45 p=0.0064\right)$. Further, the interaction between representation action and team coordination was also significant. We tested this interaction via a chi-squared test evaluating instances of team coordination with representation verses instances of team coordination without representation, for SU positive and SU negative teams. This revealed a significant relationship $\left(X^{2}=49.54 p=0.0000\right)$. 

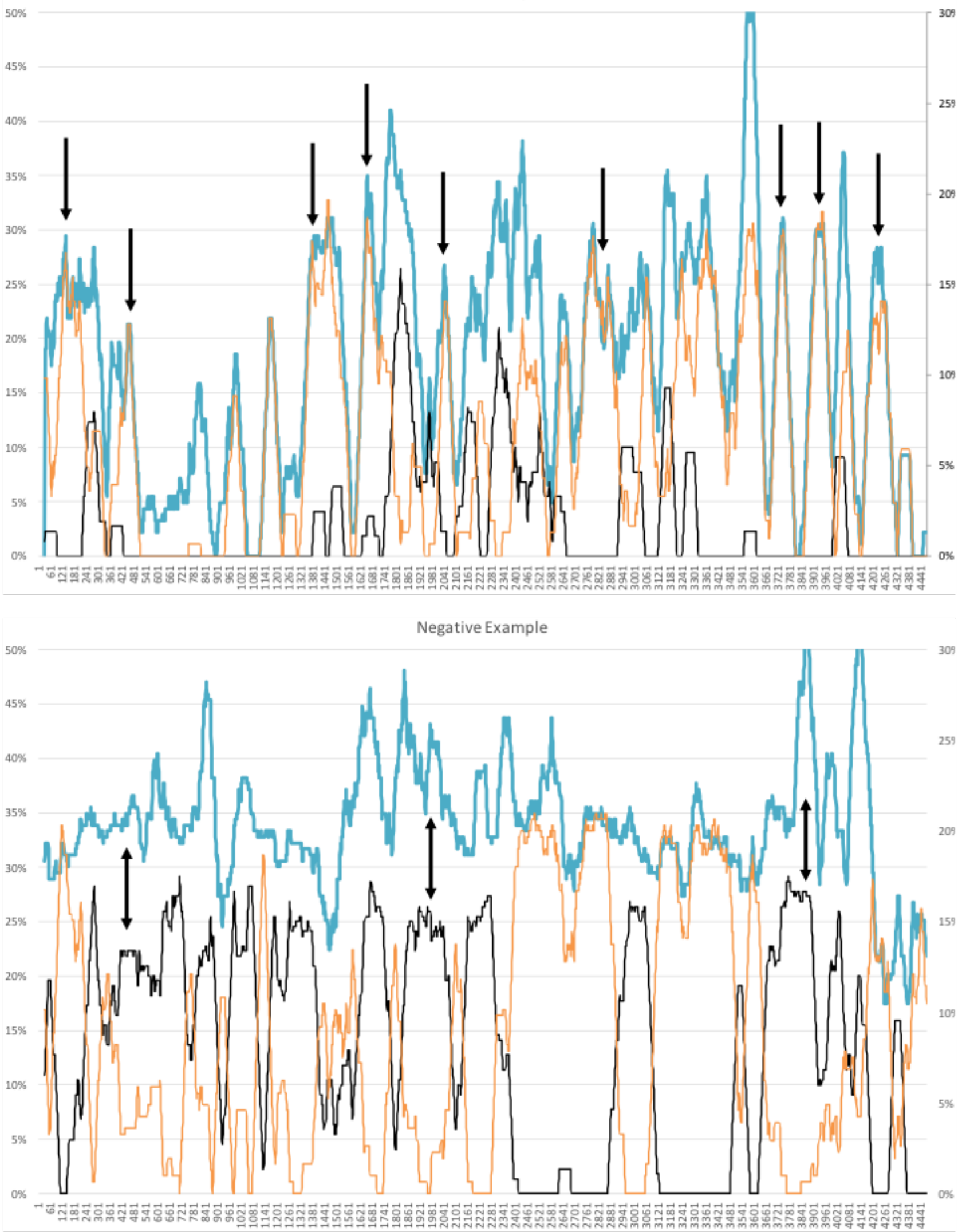

Figure 7: Action/Task level percentage intensity (left/right y-axis), time (s)(x-axis); Detail of alignment between taskwork/teamwork and action with positive: high alignment, distinct topic/action coupling / negative: low alignment, fluctuation in action and topic not linked 


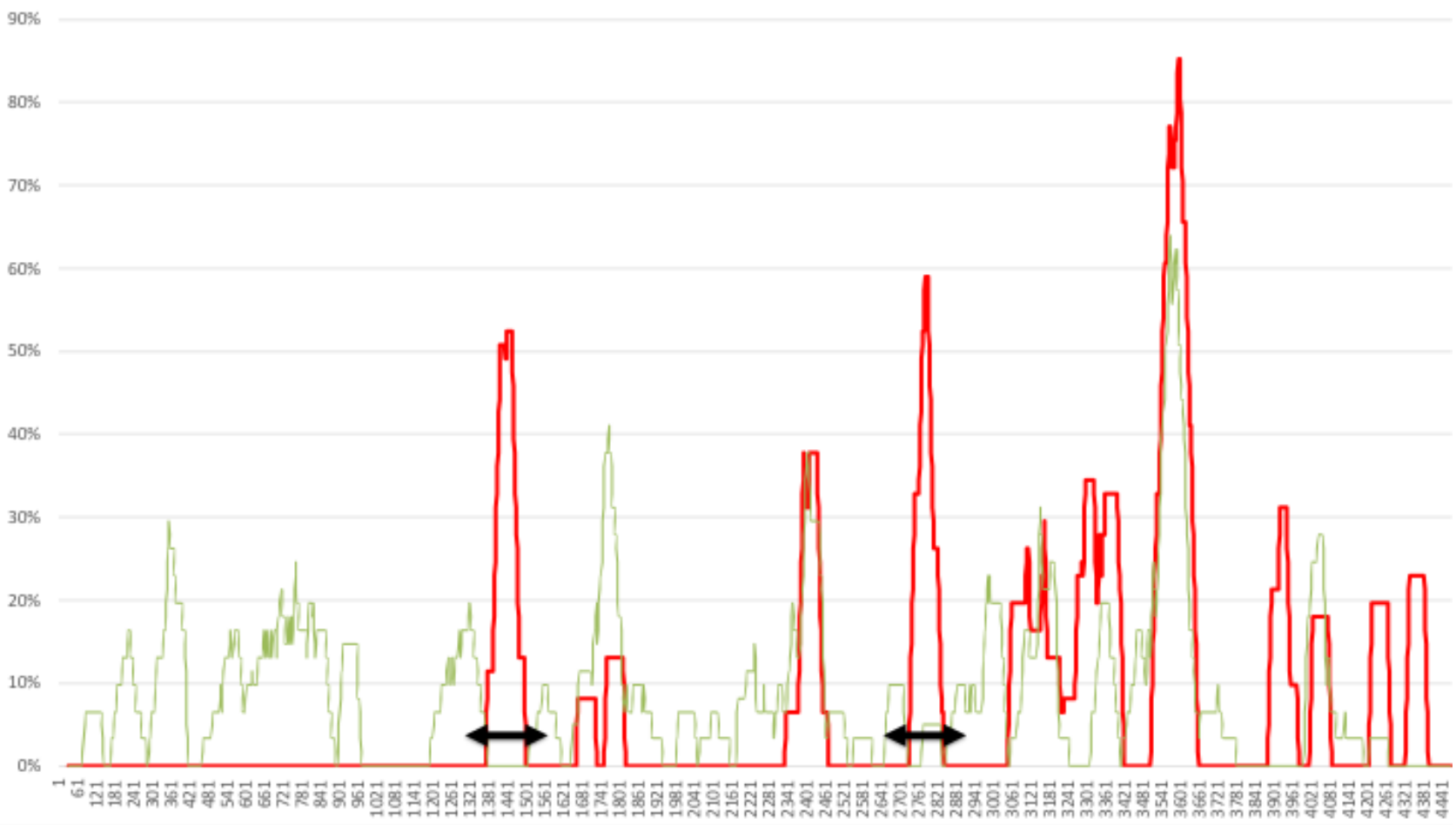

Negative Example

$100 \%$

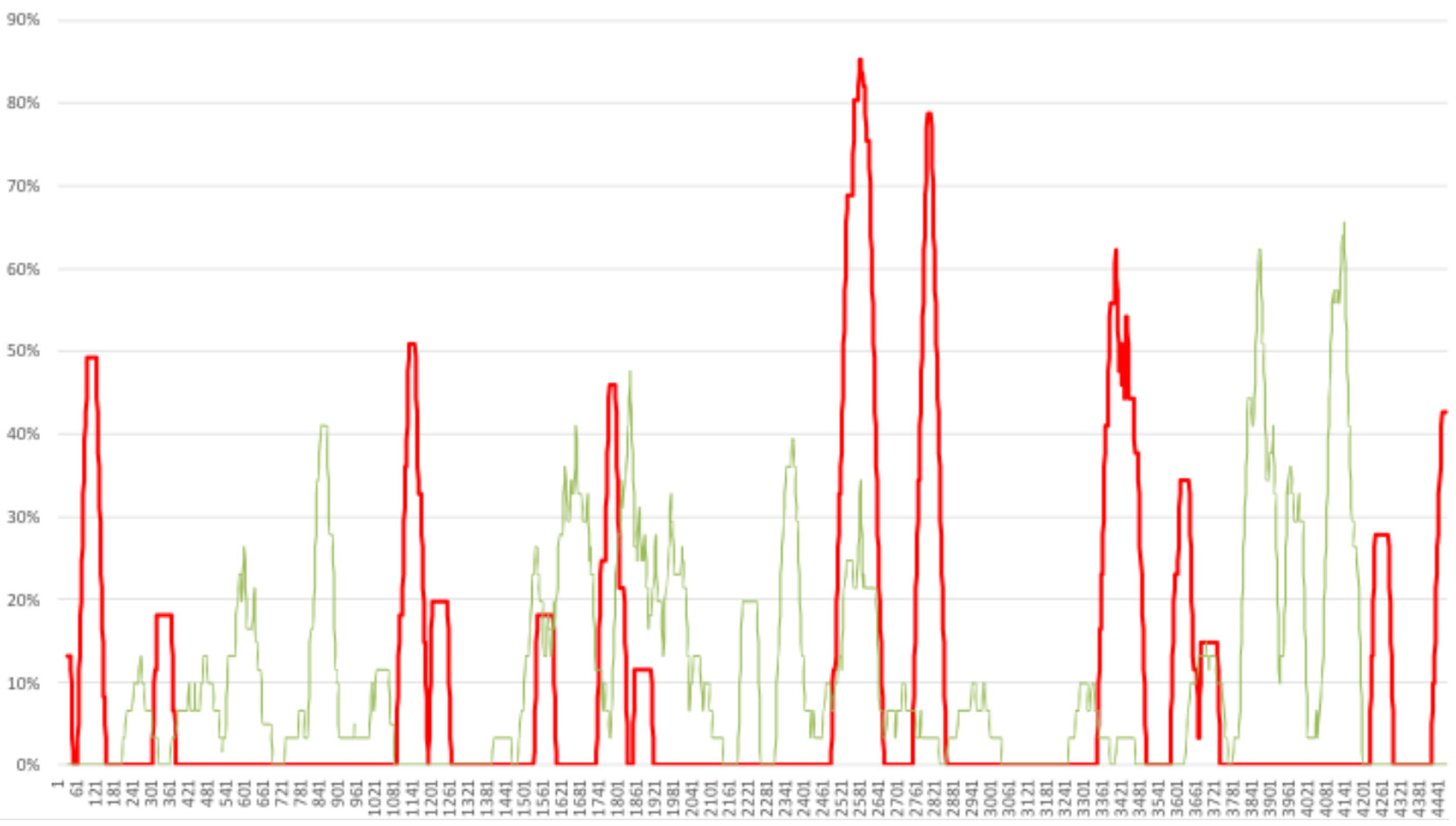

—team coordination - Representation action

Figure 8: Percentage intensity (y-axis), time (s)(x-axis); Detail of team coordination and representation action, with positive: team coordination either being aligned with representation action or being bracketed by related action (denoted by $\leftarrow \rightarrow$ )/negative: team coordination shows no direct alignment with representation action nor systematic bracketing that could be qualitatively related to the action 
The relationship between representation and team coordination can be illustrated via a qualitative example, as in Figure 9. Here, a five-minute excerpt shows a number of ways in which the task and action levels connected in a SU positive team. In the first boxed segment (Figure $9 \sim 1636-1760$ seconds) the team transitions from taskwork to teamwork. They first develop a concept sketch and reach a common understanding about the concept's mechanisms via iterative gesturing (See screen captures 1 and 2). This then forms the basis for process decision making, discussion of how to best communicate these decisions (communication process) and team coordination, resulting in a number of concluding product decisions about the concept. These are then translated into the sketch and 'prototyped' via hand gestures (Capture 3). The taskwork period is closed by extensive note taking (Capture 4). Finally, the team debates how to manage the following teamwork discussion.

The second segment (Figure 9 1776 - 1856 seconds) provides a 'bracketing' example. The team enter the design process discussion with a communication plan based on the conclusion of the first segment. During the design process discussion one team member sketches a process representation, with key links between concept and process being gestured (Capture 5). This is then used by the whole team during the coordination discussion at the end of the segment. Thus, the team coordination centres around the representation developed earlier in the discussion.

In the third segment (Figure 9 1856-1936 seconds) the team continue to discuss design process and update the sketch (Capture 6). As in the second segment, this forms the focal point for the first period of team coordination. However, the final team coordination discussion is carried out in direct connection to developments in the sketch as well as gesturing to explain changes and their implications for the team. This illustrates direct coupling between team coordination and representation action. More generally, the second and third segments illustrate bracketed interaction between representation of the design process, team coordination, further representation, and finally team coordination and representation together.

This theme connects the insights from the prior themes i.e. SU positive teams delineate their work both in terms of topic and behaviour. As such, they maintain a focus on a single aspect of the design work coupled with a single main action. As with the prior themes this allows them to resolve aspects of the problem/solution one at a time, in totality, maintaining coherence in the cognitive/behaviour approach they are taking. This helps them maintain an overview of the total information or knowledge relevant to the topic they are discussing. Similarly, SU positive teams reflectively manage switches in topic with representation action, allowing them to structure relevant information and knowledge, and cohesively link design process and design product models. As such, SU positive teams maintain continuity and coherence in terms of both topic and behaviour. Thus, while SU positive and SU negative teams deal with the same overall number of information elements SU positive teams connect, structure and align different aspects of the discussion i.e. the product to be designed and the design process needed to achieve this. 


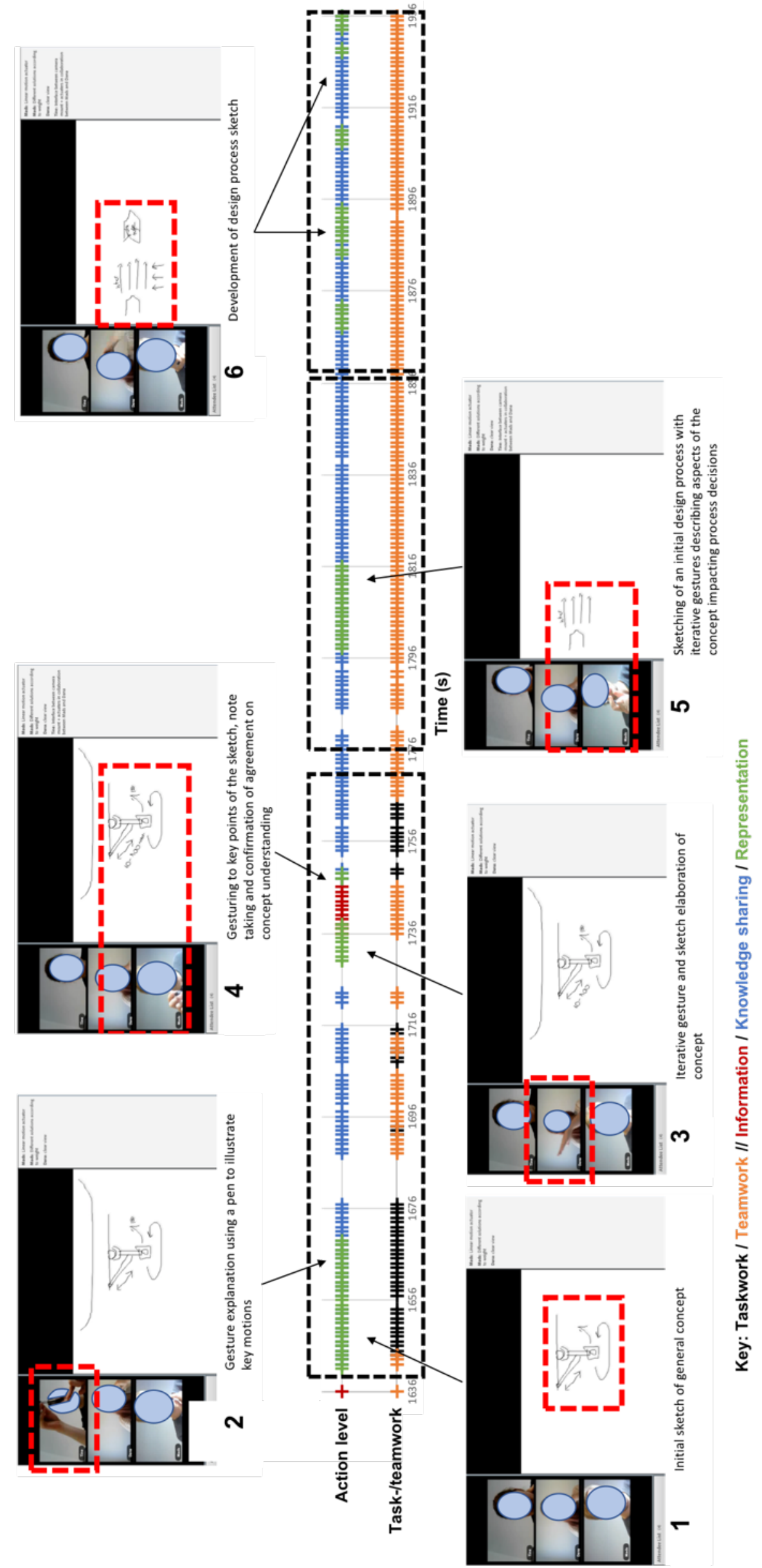

Figure 9: Detail of interaction between task and action for SU positive team 3 
Our research delivers three major insights into the impact of dynamic patterns in team processes on the development of shared understanding. These substantially extend prior empirical descriptions and provide the basis for developing theory. Throughout, we use the standard terminology and definition for moderation i.e. the magnitude (direction and/or strength) of the causal effect between two variables is dependent on the moderator (Muller, Judd, \& Yzerbyt, 2005, p. 854). First, we describe the pattern of taskwork-teamwork interdependency as a moderator of the relationship between collaborative design work and the development of shared understanding. This contrasts prior literature that has typically focused on amount of teamwork as the major determinant of shared understanding development (Hult et al., 2004; Sivasubramaniam et al., 2012). Second, we show how an inter-action 'heartbeat' pattern also moderates the relationship between collaborative design work and the development of shared understanding. Finally, we show how the impact of these patterns is moderated by cross-level pattern alignment. We distil these insights into three propositions (P1 - P3) in the conceptual model proposed in Figure 10. These propositions serve to explicitly delineate the relationships in our conceptual model, and provide the basis for testing and potential falsification in future work (Cash, 2018; Johnson, 2019; Wacker, 1998).

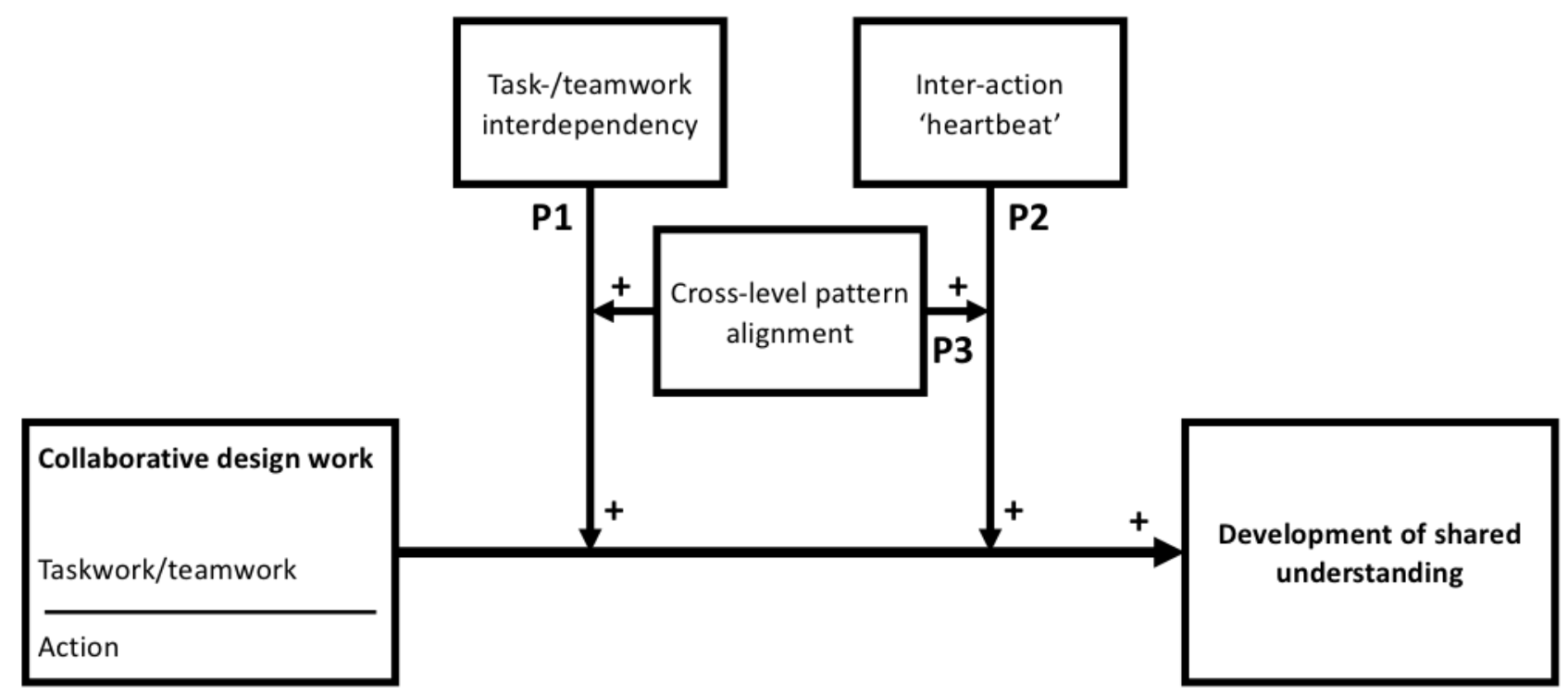

Figure 10: Proposed conceptual model describing the impact of dynamic process patterns on the development of shared understanding

First, our findings show that there is a critical taskwork-teamwork interdependence, and that progression at the task level needs to be structured in order to foster shared understanding. We define this as: separation of periods of taskwork and teamwork coupled with deliberate management of switching between taskwork and teamwork via team coordination.

Taskwork-teamwork interdependence impacts shared understanding through the alignment of product and process related goals. When goals are more defined and aligned the team is better able to structure and 
coordinate their work (Bedny \& Karwowski, 2004; Cash \& Kreye, 2018; Girard \& Robin, 2006). As the task level is sequential (Figure 1), clarity in the definition and resolution of one task and goal impacts clarity in the definition of subsequent tasks (Bedny \& Harris, 2005). Given clarity in goal, task, and resolution are all antecedents of shared understanding (Cash et al., 2017; Mulder et al., 2004; Preston et al., 2006), this finding explicitly connects task-level process patterns to shared understanding development.

This contribution builds on prior results relating to the critical interdependency between product and process. Specifically, shared understanding development has been linked to cycles of frame setting and clarification (Hey, Yu, \& Agogino, 2008); while design performance has been more generally linked to task reflexivity (De Dreu, 2007) and project structuring (Hoegl \& Weinkauf, 2005). Further, the importance of integrating perspectives has been highlighted in the contexts of virtual teams (Kanawattanachai \& Yoo, 2007) and product development (Kraaijenbrink, 2012), with critical interdependence between design process and design product models being emphasised by Girard and Robin (2006) and Badke-Schaub et al. (2007). However, our findings extend prior models, which have typically focused on the overall variance of a single factor as the determinant of shared understanding development. For example, numerous authors link the overall amount of teamwork (Pemartín et al., 2018; Sivasubramaniam et al., 2012), team coordination (Gorman, 2014; Rico, Sánchez-manzanares, Gil, \& Gibson, 2008), knowledge sharing (Dong et al., 2013; Hult et al., 2004) or question asking (Stempfle \& Badke-Schaub, 2002), in isolation, to shared understanding development. Our findings highlight the importance of deliberately managing taskwork and teamwork, in order to shape a coherent team process. We thus suggest the following proposition:

P1: The proposed pattern of taskwork-teamwork interdependency positively moderates the relationship between collaborative design work and the development of shared understanding in design teams.

Second, we describe how an inter-action 'heartbeat' pattern is a key characteristic of action level processes leading to the development of shared understanding. We define this as: separated periods of action coupled with a periodic action intensity curve.

Inter-action 'heartbeat' impacts shared understanding in two ways. First, similar to our first pattern, clarity in action-level sub-goals is essential to effective work (Bedny \& Karwowski, 2004), and can impact subsequent actions (Bedny \& Harris, 2005). Further, mixing of sub-goals leads to lack of focus on the relevant action type (Cash \& Kreye, 2017). Second, as each action is linked to a dominant cognitive process (Cash \& Kreye, 2017), periodic reduction in observed intensity allows time for cognitive knowledge construction and reflection (Aurisicchio et al., 2010; Hay, Duffy, et al., 2017), which are both antecedents of shared understanding development (Swaab, Postmes, \& Neijens, 2002). This aligns with research on knowledge sharing across whole projects, where clear, periodic patterns have been connected to performance by Maznevski and Chudoba (2000) and Massey et al. (2003). Our finding empirically demonstrates the importance of such patterns in design work, and explicitly links this to the development of shared understanding. This also 
extends prior descriptions of design team interaction (Movahed-Khah et al., 2010) and gradual sharedunderstanding development by Dong (2005).

Our second result also demonstrates how information, knowledge sharing, and representation action interact during effective design work. This extends prior research, which has typically focused on information (Deeter-Schmelz \& Ramsey, 2003; Robinson, 2010) and/or knowledge sharing (Dong et al., 2013; Markus, 2001 ) in isolation, and supports the need for integrated views of design action as suggested by, for example, Cash and Kreye's (2017) Uncertainty Driven Action model or Hay et al.'s (2017) review of design protocol studies. More specifically, our findings contextualise the importance of representation as a key element in design work that is often poorly supported by design communication tools (McAlpine, Cash, \& Hicks, 2017; Robinson, 2010; Wasiak, Hicks, Newnes, Dong, \& Burrow, 2010). Further, our results elaborate the commonly held focus on knowledge sharing as the only major factor driving the development of shared understanding in design (Carkett, 2004; Reed, Scanlan, Wills, \& Halliday, 2011; Shih, Hu, \& Chen, 2006). We found that SU negative teams displayed more knowledge sharing action (60\% of their time, in comparison to $47 \%$ in SU positive teams). As such, we conclude that overall amounts of individual actions are poor predictors for the development of shared understanding. We thus suggest the following proposition:

P2: The proposed inter-action 'heartbeat' pattern positively moderates the relationship between collaborative design work and the development of shared understanding in design teams.

Third, we show that cross-level pattern alignment (between the task level and action level) moderates the impact of patterns at these two individual levels. In addition, we propose that the alignment of team coordination and representation action occurring at inflection points in the team process support the development of shared understanding. We define this as: alignment between of periods of taskwork / teamwork and a specific action, and the deliberate linking of team coordination and representation.

Following the explanations of the prior patterns, this finding reflects clarity and alignment between goals and sub-goals across levels. It also suggests that actions are selected in conjunction with tasks. This is important because certain actions better fit specific tasks (Cash \& Kreye, 2017) e.g. information action and data organisation (Robinson, 2010) or representation action and prototyping (Sanders \& Stappers, 2014). This is similar to how frames provide context for moves in the theory reflective practice (Valkenburg \& Dorst, 1998). As such, teams displaying this pattern better align their tasks and actions across levels, facilitating the development of shared understanding as described with respect to the other two patterns in Figure 10.

This elaborates prior research, which has highlighted the general importance of overall amount of team coordination (Gorman, 2014; Rico et al., 2008), and supports Cronin and Weingart's (2007) assertion that representation is a potentially significant multiplier of coordination effort. This result connects these general managerial insights to a number of important discussions in the design literature that highlight the role of 
multi-modal representation in facilitating design negotiation (Swaab et al., 2002) and discussion (Cash \& Maier, 2016; Eris et al., 2014), as well as the link between coordination and gesture (Donovan, Heinemann, Matthews, \& Burr, 2011) or computer mediated representations that help define boundaries in which to negotiate (Brace \& Cheutet, 2012). Generally, we found that effective team coordination was connected to periods of related representation action. We thus suggest the following proposition:

P3: Cross-level pattern alignment between the taskwork-teamwork interdependency pattern and the inter-action 'heartbeat' pattern positively moderates the effect of these process patterns on the overall development of shared understanding during collaborative design work.

\subsection{Implications and Further Research}

Our findings have a number of implications for both theory and practice. First, dynamic patterns in team processes at both the task and action levels have a substantial impact on the development of shared understanding during collaborative design work. This potentially overrides prior assumptions of linear cause effect between only amount of teamwork or knowledge sharing and shared understanding development. This significantly extends theoretical models of shared understanding development and offers a number of testable propositions that could lead to further conceptual development in this area. Second, alignment between levels influences how process patterns at any one level impact overall shared understanding development. This confronts the general conceptual separation of theory, research, and literature dealing with the different levels of activity and perspectives on team processes (teamwork and taskwork), and points to the need for a synergistic view of design work. Finally, we highlight a number of specific patterns of interaction between team coordination and representation that offer the potential for substantial further exploration.

In terms of practice our findings offer three actionable insights. First, design teams should be encouraged to draw up meta-models that link their product and process (e.g. diagrams which link the output from their taskwork and teamwork via mindmaps or cause-effect chains). Such meta-models should be used to deliberately manage progression between teamwork and taskwork related topics of discussion. Second, design teams should be encouraged to deliberately structure their combination of information, knowledge sharing and representation actions, following a 'heartbeat' where actions are distinctly separated, and time is allowed for reflection, particularly at transition points between taskwork and teamwork. Teams should maintain a focus on a single aspect of the design work coupled with a single main action, resolving aspects of the problem/solution one at a time. Finally, while team coordination is generally important, its effectiveness is dependent on its position in the process and its representational support. Design teams should be encouraged to focus coordination effort at transition points between taskwork and teamwork, and to support this with representation e.g. by drawing and expanding a meta-model that captures the interface between product and process. 
Finally, our work points to a number of avenues of further research beyond the examination of the propositions. First, the studied teams were small. A number of researchers have highlighted the potential impact of team network structure (Braha, 2016; Leenders \& Dolfsma, 2016) and process alignment (Crawford \& Lepine, 2013; Movahed-Khah et al., 2010) on dynamic process effects. Thus, further study of meso level large team and team/inter-team processes over longer time periods would substantially extend current knowledge. Second, the real time identification of and reflection on dynamic patterns in team processes, is little explored. Our findings extend beyond Schön's $(1983,1987)$ theory of reflection in practice to suggest that reflective patterns in teams could lead to greater shared understanding. Thus, there is potential for research taking a starting point in reflective practice (Valkenburg \& Dorst, 1998) or process awareness (Valgeirsdottir \& Onarheim, 2017) to be adapted into the team process context, particularly leveraging approaches to theorising from process data (Langley, 1999).

\section{$5 \quad$ Conclusions and Further Research}

This paper explored how dynamic patterns in team processes impact the development of shared understanding during design work. Based on an exploratory, in-depth comparative study we develop novel theoretical propositions that suggest how dynamic patterns in team processes can have a substantial moderating effect on the development of shared understanding in design. First, we propose a pattern of 'taskwork-teamwork interdependency'. Second, we characterise a rhythmic 'inter-action heartbeat' pattern. We describe how both of these patterns appear to moderate the relationship between collaborative design work and the development of shared understanding. Third, we propose that 'cross-level pattern alignment' between these two patterns moderates their effect. Finally, we show how this cross-level alignment is supported by linked team coordination and representation action. We synthesise these insights into a conceptual model and propositions with implications for design process theory, as well as design practitioners. Importantly, our results begin to question the general assumption for shared understanding development that 'more is better' with respect to teamwork, team coordination, and knowledge sharing. Rather, we show that the dynamic patterns in team processes identified in this work critically impact the relationship between collaborative design work and shared understanding development.

\section{References}

Ahmed, S. (2005). Encouraging reuse of design knowledge: A method to index knowledge. Design Studies, 26(6), 565-592.

Ahmed, S., \& Storga, M. (2009). Merged ontology for engineering design: Contrasting empirical and theoretical approaches to develop engineering ontologies. Al EDAM (Artificial Intelligence for Engineering Design, Analysis and Manufacturing), 23(4), 391-407.

Ariff, N. S. N. A., Eris, O., \& Badke-Schaub, P. (2013). How Designers Express Agreement. In 5th IASDR Conference (pp. 1-10). Tokyo, Japan.

Aurisicchio, M., Ahmed, S., \& Wallace, K. (2007). Questions as a tool to design. In ASME Conference on Design Theory and Methodology. Las Vegas, USA.

Aurisicchio, M., Bracewell, R., \& Wallace, K. (2010). Understanding how the information requests of aerospace engineering designers influence information-seeking behaviour. Journal of Engineering 
Design, 21(6), 707-730.

Badke-Schaub, P., Lauche, K., Neumann, A., \& Ahmed, S. (2007). Task - Team - Process: Assessment and Analysis of the Development of Shared Representations in an Engineering Team. In Design Thinking Research Symposium (pp. 1-12). London, UK.

Bedny, G. Z., \& Harris, S. R. (2005). The systemic-structural theory of activity: Applications to the study of human work. Mind, Culture, and Activity, 12(2), 128-147.

Bedny, G. Z., \& Karwowski, W. (2004). Activity theory as a basis for the study of work. Ergonomics, 47(2), 134-153.

Brace, W., \& Cheutet, V. (2012). A framework to support requirements analysis in engineering design. Journal of Engineering Design, 23(12), 873-901.

Braha, D. (2016). The Complexity of Design Networks: Structure and Dynamics. In P. Cash, T. Stankovic, \& M. Storga (Eds.), Experimental Design Research: Approaches, perspectives, applications (pp. 129-151). Springer.

Carkett, R. (2004). “He's different, he's got 'Star Trek' Vision": Supporting the expertise of conceptual design engineers. Design Studies, 25(5), 459-475.

Cash, P. (2018). Developing theory-driven design research. Design Studies, 56(May), 84-119.

Cash, P., Dekoninck, E., \& Ahmed-Kristensen, S. (2017). Supporting the development of shared understanding in distributed design teams. Journal of Engineering Design, 28(3), 147-170.

Cash, P., \& Kreye, M. E. (2017). Uncertainty Driven Action (UDA) model: A foundation for unifying perspectives on design activity. Design Science, 3(e26), 1-41.

Cash, P., \& Kreye, M. E. (2018). Exploring Uncertainty Perception as a Driver of Design Activity. Design Studies, 54(January), 50-79.

Cash, P., \& Maier, A. (2016). Prototyping with your hands: the many roles of gesture in the communication of design concepts. Journal of Engineering Design, 27(1-3), 118-145.

Chiu, C.-M., Hsu, M.-H., \& Wang, E. T. G. (2006). Understanding knowledge sharing in virtual communities: An integration of social capital and social cognitive theories. Decision Support Systems, 42(3), 18721888.

Christensen, L. B., Johnson, R. B., \& Turner, L. A. (2011). Research Methods, Design, and Analysis (11th ed.). Boston, USA: Pearson.

Crawford, E. R., \& Lepine, J. A. (2013). A configural theory of team processes: Accounting for the structure of taskwork and teamwork. Academy of Management Review, 38(1), 32-48.

Cronin, M. A., \& Weingart, L. R. (2007). Representational gaps, information processing, and conflict in functionally diverse teams. Academy of Management Journal, 32(3), 761-773.

De Dreu, C. K. W. (2007). Cooperative outcome interdependence, task reflexivity, and team effectiveness: A motivated information processing perspective. Journal of Applied Psychology, 92(3), 628-638.

De Dreu, C. K. W., Nijstad, B. A., \& Van Knippenberg, D. (2008). Motivated information processing in group judgment and decision making. Personality and Social Psychology Review, 12(1), 22-49.

Deeter-Schmelz, D. R., \& Ramsey, R. P. (2003). An Investigation of Team Information Processing in Service Teams: Exploring the Link between Teams and Customers. Journal of the Academy of Marketing Science, 31(4), 409-424.

Deken, F., Kleinsmann, M., Aurisicchio, M., Lauche, K., \& Bracewell, R. (2012). Tapping into past design experiences: Knowledge sharing and creation during novice-expert design consultations. Research in Engineering Design, 23(3), 203-218.

Dong, A. (2005). The latent semantic approach to studying design team communication. Design Studies, 26(5), 445-461.

Dong, A., Kleinsmann, M., \& Deken, F. (2013). Investigating design cognition in the construction and enactment of team mental models. Design Studies, 34(1), 1-33.

Donovan, J., Heinemann, T., Matthews, B., \& Burr, J. (2011). Getting the point: The role of gesture in managing intersubjectivity in a design activity. Artificial Intelligence for Engineering Design, Analysis and Manufacturing, 25(3), 221-235.

Eisenhardt, K. M., \& Graebner, M. E. (2007). Theory building from cases: Opportunities and challenges. Academy of Management Journal, 50(1), 25-32.

Eris, O. (2002). Perceiving, comprehending and measuring design activity through the questions asked while designing. Stanford, Mechanical Engineering. 
Eris, O., Martelaro, N., \& Badke-Schaub, P. (2014). A comparative analysis of multimodal communication during design sketching in co-located and distributed environments. Design Studies, 35(6), 559-592.

Espinosa, J., Slaughter, S., Kraut, R., \& Herbsleb, J. (2007). Team Knowledge and Coordination in Geographically Distributed Software Development. Journal of Management Information Systems, 24(1), 135-169.

Garcia, R. (2005). Uses of agent-based modeling in innovation/new product development research. Journal of Product Innovation Management, 22(5), 380-398.

Gero, J. S., \& Mc Neill, T. (1998). An approach to the analysis of design protocols. Design Studies, 19(1), 2161.

Gilson, L. L., Maynard, M. T., Jones Young, N. C., Vartiainen, M., \& Hakonen, M. (2015). Virtual Teams Research: 10 Years, 10 Themes, and 10 Opportunities. Journal of Management, 41(5), 1313-1337.

Girard, P., \& Robin, V. (2006). Analysis of collaboration for project design management. Computers in Industry, 57(8-9), 817-826.

Gorman, J. C. (2014). Team Coordination and Dynamics: Two Central Issues. Current Directions in Psychological Science, 23(5), 355-360.

Gorman, J. C., Amazeen, P. G., \& Cooke, N. J. (2010). Team Coordination Dynamics. Nonlinear Dynamics Psychology and Life Sciences, 14(3), 265-289.

Grossman, R., Friedman, S., \& Kalra, S. (2017). Teamwork processes and emergent states. In E. Salas, R. Rico, \& J. Passmore (Eds.), The wiley blackwell handbook of the psychology of team working and collaborative processes (1st ed., pp. 245-269). John Wiley \& Sons.

Handfield, R., \& Melnyk, S. A. (1998). The scientific theory-building process: a primer using the case of TQM. Journal of Operations Management, 16(4), 321-339.

Hay, L., Duffy, A., McTeague, C., Pidgeon, L., Vuletic, T., \& Grealy, M. (2017). A systematic review of protocol studies on conceptual design cognition: Design as search and exploration. Design Science, 3(Visser 2004), e10.

Hay, L., McTeague, C., Duffy, A., Pidgeon, L., Vuletic, T., \& Grealy, M. (2017). A Systematic Review of Protocol Studies on Conceptual Design Cognition. In Design Computing and Cognition '16 (pp. 135-153). Cham: Springer International Publishing.

Hey, J., Yu, J., \& Agogino, A. M. (2008). Design team framing: Paths and principles. In Proceedings of the ASME Design Engineering Technical Conference (pp. 409-420).

Hinds, P. J., \& Mortensen, M. (2005). Understanding Conflict in Geographically Distributed Teams: The Moderating Effects of Shared Identity, Shared Context, and Spontaneous Communication. Organization Science, 16(3), 290-307.

Hoegl, M., \& Weinkauf, K. (2005). Managing task interdependencies in multi-team projects: A longitudinal study. Journal of Management Studies, 42(6), 1287-1308.

Hult, G. T. M., Ketchen, D. J., \& Slater, S. F. (2004). Information processing, knowledge development, and strategic supply chain performance. Academy of Management Journal, 47(2), 241-253.

Humayun, M., \& Gang, C. (2013). An Empirical Study on Improving Shared Understanding of Requirements in GSD. International Journal of Software Engineering and Its Applications, 7(1), 79-92.

Johnson, J. S. (2019). Developing qualitative propositions in sales research: existing approaches and a new multiphasic technique. Journal of Personal Selling and Sales Management, $0(0), 1-6$.

Johnson, T. E., Lee, Y., Lee, M., O'Connor, D. L., Khalil, M. K., \& Huang, X. (2007). Measuring Sharedness of Team-Related Knowledge: Design and Validation of a Shared Mental Model Instrument. Human Resource Development International, 10(4), 437-454.

Johnson, T. E., \& O'Connor, D. L. (2008). Measuring Team Shared Understanding Using the AnalysisConstructed Shared Mental Model Methodology. Performance Improvement Quarterly, 21(3), 113-134.

Kanawattanachai, P., \& Yoo, Y. (2007). The impact of knowledge coordination on virtual team performance over time. Mis Quarterly, 31(4), 783-808.

Kaptelinin, V. (2005). The object of activity: Making sense of the sense-maker. Mind, Culture, and Activity, 12(1), 4-18.

Ko, D.-G., Kirsch, L. J., \& King, W. R. (2005). Antecedents of knowladge transfer from consultants to clients in enterprise system implementations. MIS Quarterly, 29(1), 59-85.

Kozlowski, S. W. J., \& Ilgen, D. R. (2006). Enhancing the effectiveness of work groups and teams. Psychological Science in the Public Interest, 7(3), 77-124. 
Kraaijenbrink, J. (2012). Integrating knowledge and knowledge processes: A critical incident study of product development projects. Journal of Product Innovation Management, 29(6), 1082-1096.

Krippendorff, K. (1981). Content analysis: An introduction to its methodology. (F. G. Kline \& S. H. Evans, Eds.)The Sage CommText Series (Vol. Second). Thousand Oaks, CA, CA, USA: Sage.

Langley, A. (1999). Strategies for Theorizing from Process Data. The Academy of Management Review, 24(4), 691-710.

Leenders, R., Contractor, N. S., \& DeChurch, L. A. (2016). Once upon a time: Understanding team processes as relational event networks. Organizational Psychology Review, 6(1), 92-115.

Leenders, R., \& Dolfsma, W. A. (2016). Social Networks for Innovation and New Product Development. Journal of Product Innovation Management, 33(2), 123-131.

Lehmann-Willenbrock, N., \& Allen, J. A. (2018). Modeling Temporal Interaction Dynamics in Organizational Settings. Journal of Business and Psychology, 33(3), 325-344.

Liu, J., Chen, J., \& Tao, Y. (2015). Innovation performance in new product development teams in China's technology ventures: The role of behavioral integration dimensions and collective efficacy. Journal of Product Innovation Management, 32(1), 29-44.

Marks, M. A., Mathieu, J. E., \& Zaccaro, S. J. (2001). A temporally based framework and taxonomy of team processes. Academy of Management Review, 26(3), 356-376.

Markus, L. M. (2001). Toward a Theory of Knowledge Reuse: Types of Knowledge Reuse Situations and Factors in Reuse Success. Journal of Management Information Systems, 18(1), 57-93.

Massey, A. P., Montoya-weiss, M. M., \& Hung, Y.-T. (2003). Because Time Matters: Temporal Coordination in Global Virtual Project Teams. Journal of Management Information Systems, 19(4), 129-155.

Mathieu, J. E., Heffner, T. S., Goodwin, G., Salas, E., \& Cannon-Bowers, J. (2000). The Influence of Shared Mental Models on Team Process and Performance. Journal of Applied Psychology, 85(2), 273-283.

Maznevski, M. L., \& Chudoba, K. M. (2000). Bridging Space Over Time: Global Virtual Team Dynamics and Effectiveness. Organization Science, 11(5), 473-492.

McAlpine, H., Cash, P., \& Hicks, B. (2017). The role of logbooks as mediators of engineering design work. Design Studies, 48(January), 1-29.

Movahed-Khah, R., Ostrosi, E., \& Garro, O. (2010). Analysis of interaction dynamics in collaborative and distributed design process. Computers in Industry, 61(1), 2-14.

Mulder, I., Swaak, J., \& Kessels, J. (2002). Assessing group learning and shared understanding in technologymediated interaction. Journal of Educational Technology \& Society, 5(1), 35-47.

Mulder, I., Swaak, J., \& Kessels, J. (2004). In search of reflective behavior and shared understanding in ad hoc expert teams. Cyberpsychology \& Behavior, 7(2), 141-54.

Muller, D., Judd, C. M., \& Yzerbyt, V. Y. (2005). When moderation is mediated and mediation is moderated. Journal of Personality and Social Psychology, 89(6), 852-863.

Navarro, J., Roe, R. A., \& Artiles, M. I. (2015). Taking time seriously: Changing practices and perspectives in Work/Organizational Psychology. Journal of Work and Organizational Psychology, 31(3), 135-145.

Neuendorf, K. A. (2017). The content analysis guidebook (2nd ed.). Thousand Oaks, USA: SAGE Publications, Incorporated.

Neuman, L. (1997). Social research methods: Qualitative and quantitative approaches. Boston, USA: Allyn and Bacon.

Paletz, S. B. F., Chan, J., \& Schunn, C. D. (2017). The dynamics of micro-conflicts and uncertainty in successful and unsuccessful design teams. Design Studies, 50, 39-69.

Pask, G. (1975). Conversation, cognition and learning. New York: Elsevier.

Pemartín, M., Rodríguez-Escudero, A. I., \& Munuera-Alemán, J. L. (2018). Effects of Collaborative Communication on NPD Collaboration Results: Two Routes of Influence. Journal of Product Innovation Management, 35(2), 184-208.

Preston, D. S., Karahanna, E., \& Rowe, F. (2006). Development of shared understanding between the Chief Information officer and top management team in U.S. and French Organizations: a cross-cultural comparison. IEEE Transactions on Engineering Management, 53(2), 191-206.

Qu, Y., \& Hansen, D. L. (2008). Building Shared Understanding in Collaborative Sensemaking. In Proceedings of the Workshop on Sensemaking at the Conference on Human Factors in Computing Systems (CHI) (pp. 1-5). Florence, Italy.

Reed, N., Scanlan, J., Wills, G., \& Halliday, S. T. (2011). Knowledge use in an advanced manufacturing 
environment. Design Studies, 32(3), 292-312.

Reimer, T., Park, E. S., \& Hinsz, V. B. (2006). Shared and coordinated cognition in competitive and dynamic task environments: An information-processing perspective for team sports. International Journal of Sport and Exercise Psychology, 4(4), 376-400.

Reiter-Palmon, R., Sinha, T., Gevers, J., Odobez, J. M., \& Volpe, G. (2017). Theories and Models of Teams and Groups. Small Group Research, 48(5), 544-567.

Rico, R., Sánchez-manzanares, M., Gil, F., \& Gibson, C. (2008). Team Implicit Coordination Processes: A Team Knowledge-Based Approach. Academy of Management Review, 33(1), 163-184.

Robinson, M. A. (2010). An empirical analysis of engineers' information behaviours. Journal of the American Society for Information Science and Technology, 61(4), 640-658.

Robson, C., \& McCartan, K. (2011). Real world research (4th ed.). Chichester: Wiley.

Sanders, E. B. N., \& Stappers, P. J. (2014). Probes, toolkits and prototypes: three approaches to making in codesigning. CoDesign, 10(1), 5-14.

Sarin, S., \& O'Connor, G. C. (2009). First among equals: The effect of team leader characteristics on the internal dynamics of cross-functional product development teams. Journal of Product Innovation Management, 26(2), 188-205.

Schön, D. A. (1983). The reflective practitioner: How professionals think in action. New York, USA: Harper Torchbooks.

Schön, D. A. (1987). Educating the reflective practitioner. San Francisco: Jossey-Bass.

Shadish, W. R., Cook, T. D., \& Campbell, D. T. (2002). Experimental and quasi-experimental designs for generalized causal inference. Boston, US: Mifflin and Company.

Shih, S. G., Hu, T. P., \& Chen, C. N. (2006). A game theory-based approach to the analysis of cooperative learning in design studios. Design Studies, 27(6), 711-722.

Shroyer, K., Lovins, T., Turns, J., Cardella, M. E., \& Atman, C. J. (2018). Timescales and ideaspace: An examination of idea generation in design practice. Design Studies, 57, 9-36.

Sim, S. K., \& Duffy, A. H. B. (2003). Towards an ontology of generic engineering design activities. Research in Engineering Design, 14(4), 200-223.

Sivasubramaniam, N., Liebowitz, S. J., \& Lackman, C. L. (2012). Determinants of new product development team performance:A meta-analytic review. Journal of Product Innovation Management, 29(5), 803820.

Stempfle, J., \& Badke-Schaub, P. (2002). Thinking in design teams - an analysis of team communication. Design Studies, 23(5), 473-496.

Storga, M., Andreasen, M. M., \& Marjanovic, D. (2010). The design ontology: Foundation for the design knowledge exchange and management. Journal of Engineering Design, 21(4), 427-454.

Swaab, R. I., Postmes, T., \& Neijens, P. (2002). Multiparty negotiation support: The role of visualization's influence on the development of shared mental models. Journal of Management Information Systems, 19(1), 129-150.

Taylor, T. P., \& Ahmed-Kristensen, S. (2018). Global product development projects: measuring performance and monitoring the risks. Production Planning and Control, 29(15), 1290-1302.

Valgeirsdottir, D., \& Onarheim, B. (2017). Metacognition in Creativity: Process Awareness Used to Facilitate the Creative Process. In B. T. Christensen \& L. J. Ball (Eds.), Analysing Design Thinking: Studies of CrossCultural Co-Creation (pp. 215-228). London, UK: CRC Press.

Valkenburg, R., \& Dorst, K. (1998). The reflective practice of design teams. Design Studies, 19(3), 249-271.

Wacker, J. G. (1998). A definition of theory: Research guidelines for different theory-building research methods in operations management. Journal of Operations Management, 16(4), 361-385.

Wasiak, J., Hicks, B. J., Newnes, L., Dong, A., \& Burrow, L. (2010). Understanding engineering email: the development of a taxonomy for identifying and classifying engineering work. Research in Engineering Design, 21(1), 43-64.

Wiltschnig, S., Christensen, B. T., \& Ball, L. J. (2013). Collaborative problem-solution co-evolution in creative design. Design Studies, 34(5), 515-542.

Yin, R. K. (2013). Case study research: Design and methods. Thousand Oaks, CA, USA: Sage Publications. 
Appendix A: Raw correlation data for key variables

\begin{tabular}{|c|c|c|c|c|c|c|c|c|c|}
\hline \multirow[t]{2}{*}{ Team } & & \multicolumn{4}{|c|}{ SU Positive } & \multicolumn{4}{|c|}{ SU Negative } \\
\hline & & 1 & 2 & 3 & 4 & 1 & 2 & 3 & 4 \\
\hline \multirow{2}{*}{$\begin{array}{l}\text { Dependant } \\
\text { variables }\end{array}$} & $\%$ Increase in SU & 40.4 & 19.3 & 21.1 & 14.3 & -12.8 & -10.3 & -23.5 & -14.5 \\
\hline & $\%$ Decrease in concepts & -25.9 & -20.0 & 11.1 & -5.4 & 14.5 & 4.2 & -12.9 & 12.3 \\
\hline \multirow{9}{*}{$\begin{array}{l}\text { Total time } \\
\text { (Seconds) }\end{array}$} & Team coordination & 290 & 453 & 575 & 234 & 365 & 414 & 381 & 803 \\
\hline & Teamwork & 264 & 461 & 324 & 379 & 335 & 480 & 314 & 429 \\
\hline & Taskwork & 72 & 40 & 291 & 86 & 330 & 60 & 231 & 229 \\
\hline & Taskwork/Teamwork overlap & 201 & 134 & 454 & 248 & 509 & 195 & 372 & 500 \\
\hline & Information action & 317 & 598 & 471 & 493 & 539 & 1035 & 653 & 537 \\
\hline & Knowledge sharing action & 1525 & 1970 & 2924 & 2061 & 3222 & 2223 & 2346 & 3048 \\
\hline & Representation action & 233 & 419 & 539 & 231 & 307 & 602 & 286 & 436 \\
\hline & Action overlap & 879 & 1607 & 1386 & 1065 & 1873 & 2823 & 1742 & 1744 \\
\hline & Action intensity & 2076 & 2986 & 3935 & 2784 & 4068 & 3861 & 3286 & 4021 \\
\hline
\end{tabular}


SU Positive 1
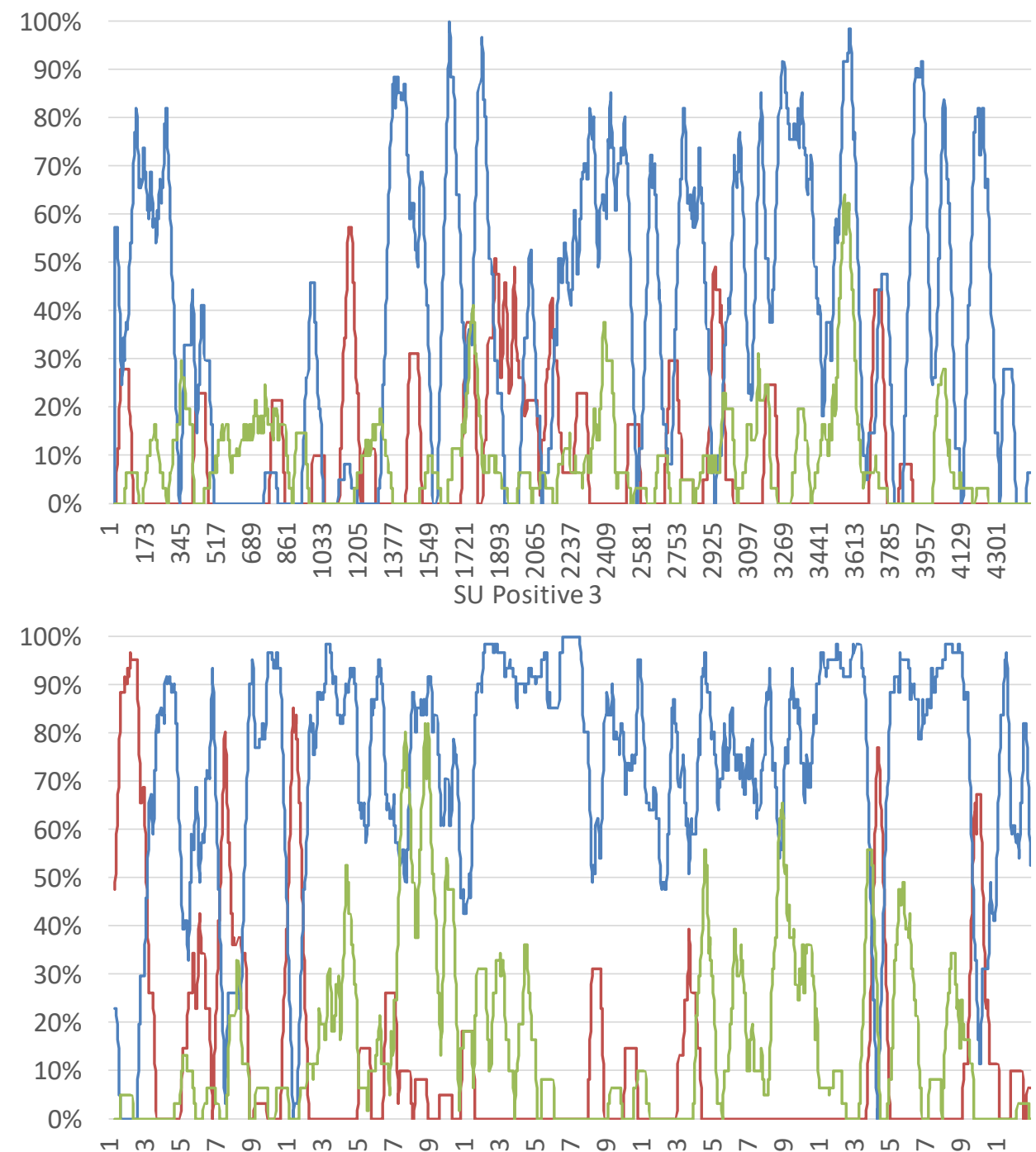

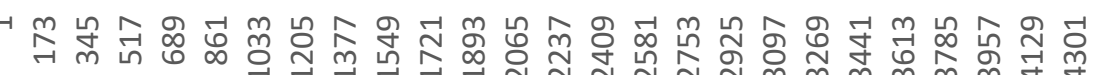

SU Positive 2
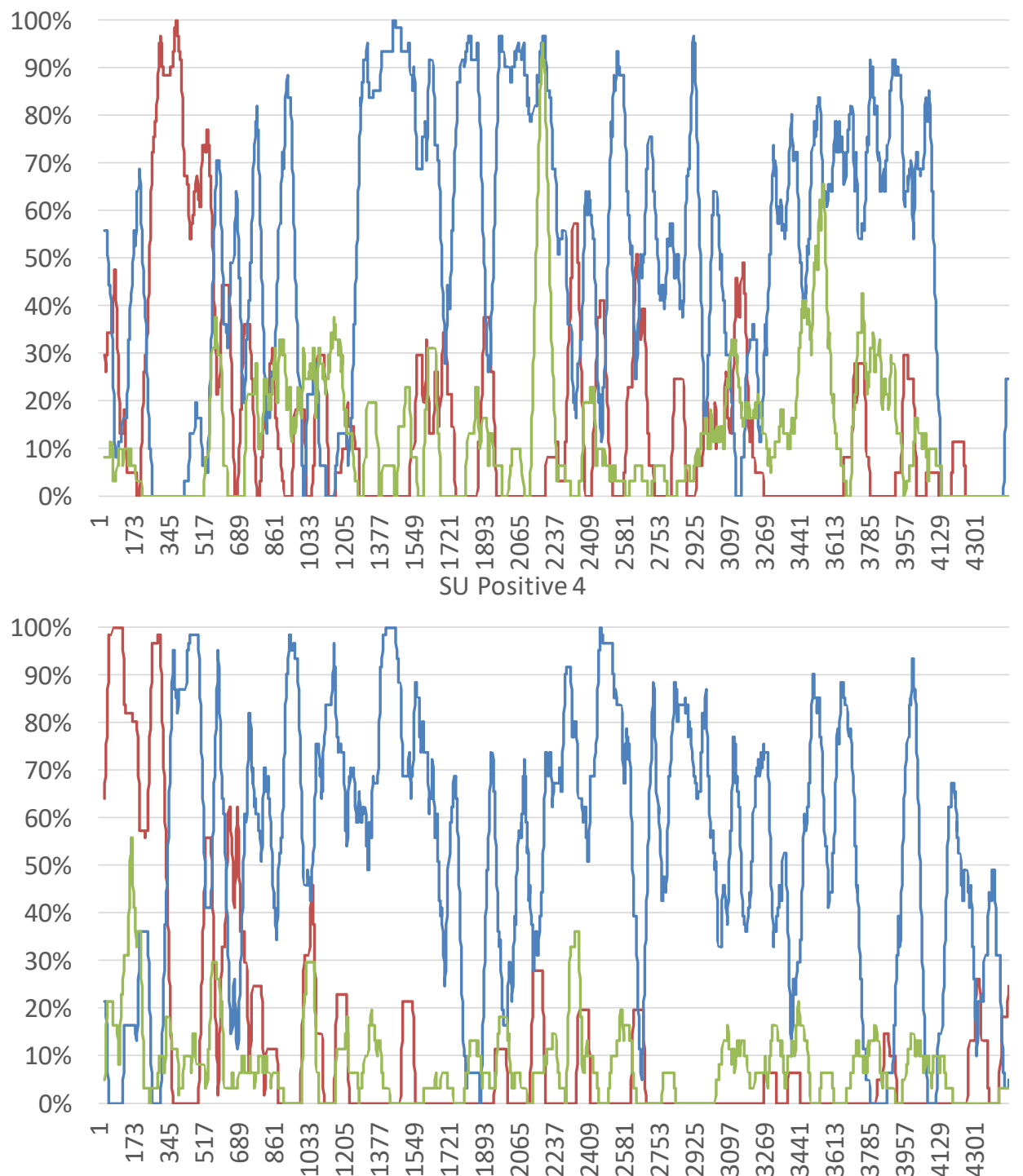

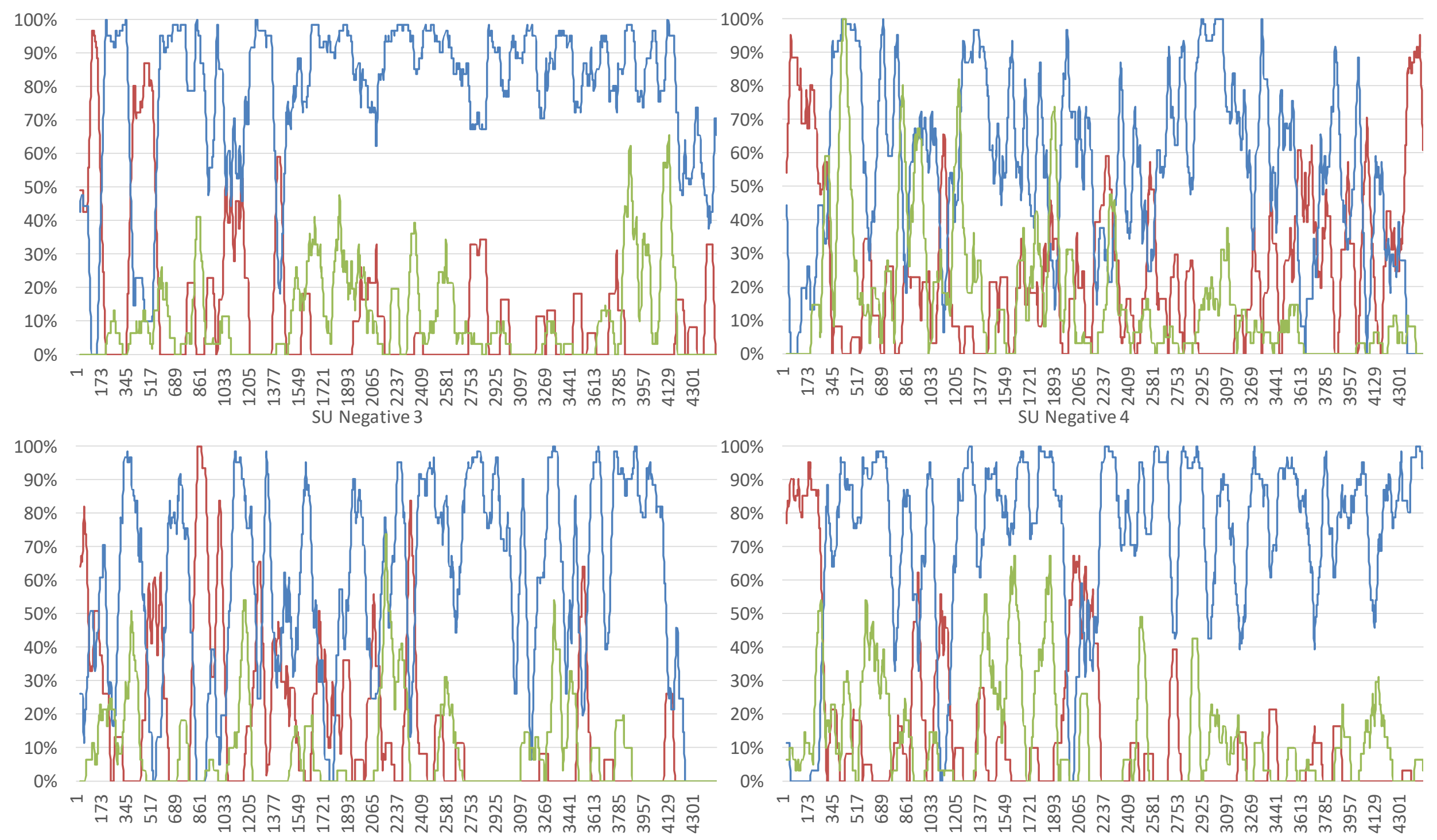
Appendix C: Action/Task level percentage intensity (left/right y-axis), time (s)(x-axis); Detail of alignment between taskwork/teamwork and action for all teams

SU Positive 1

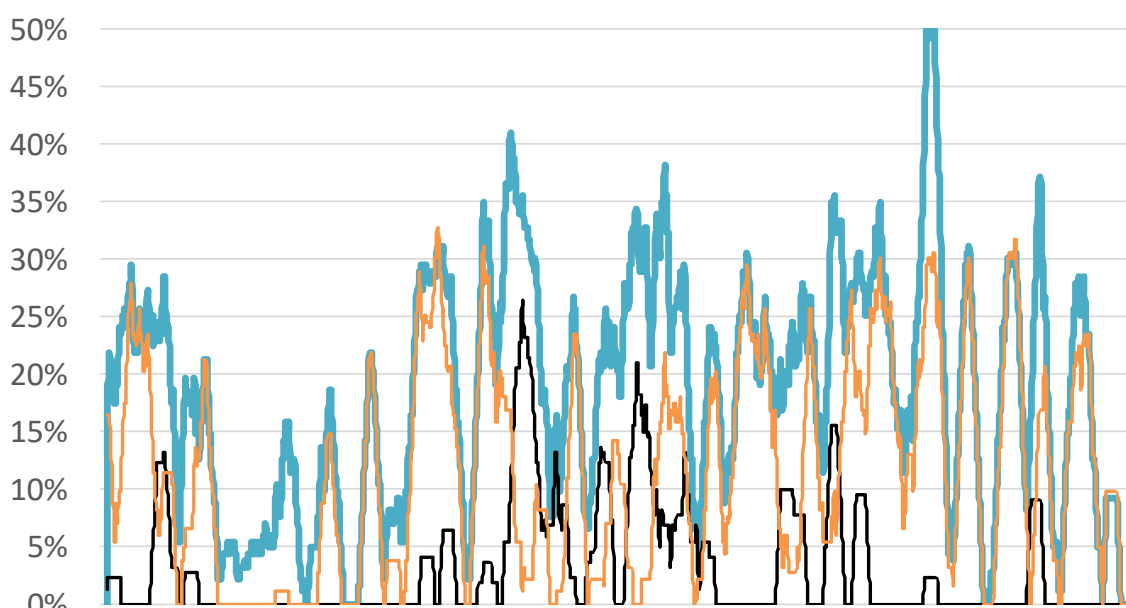

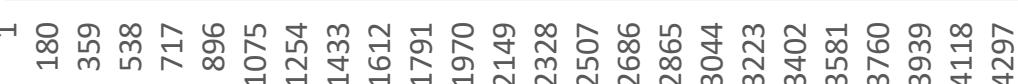

\section{SU Positive 3}

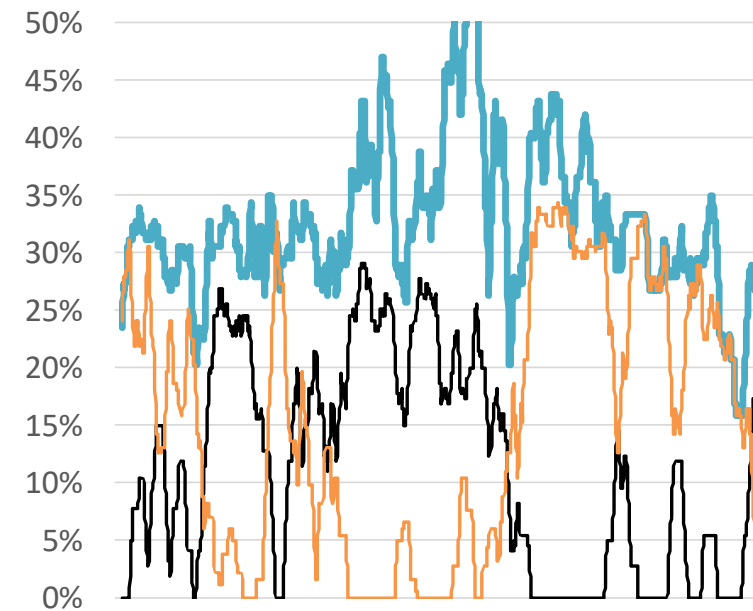

ᄀ \&
SU Positive 2

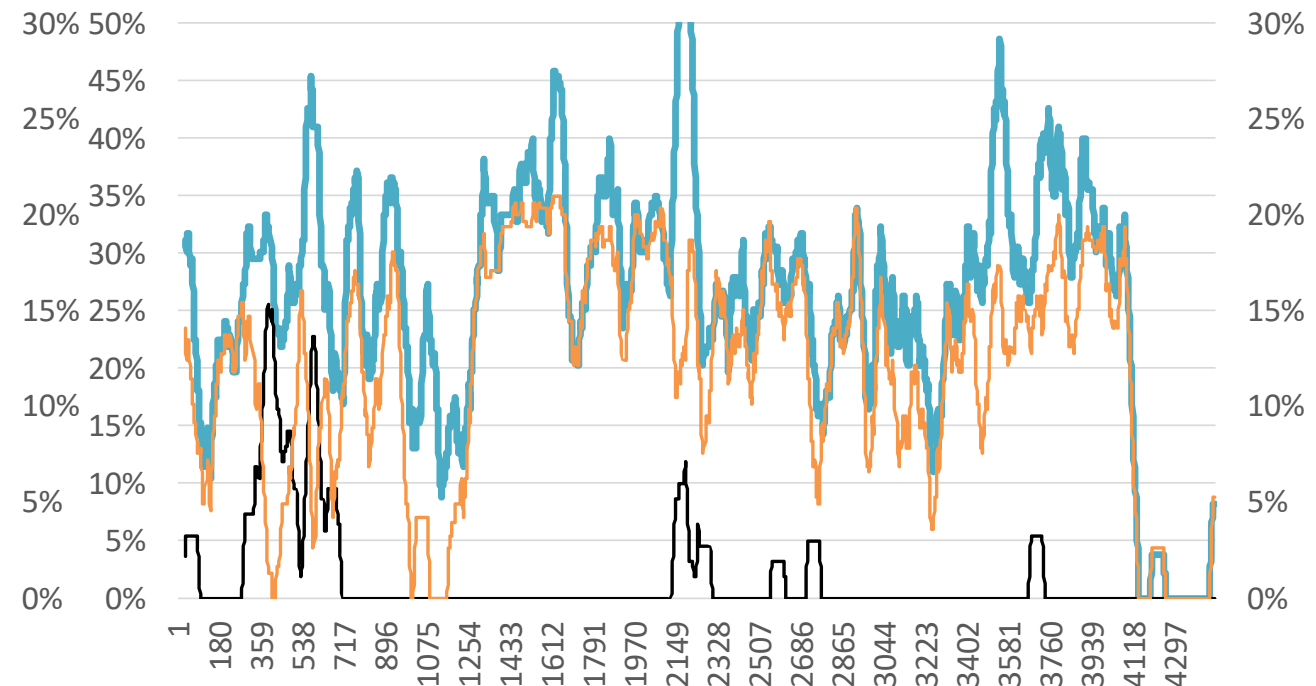

SU Positive 4

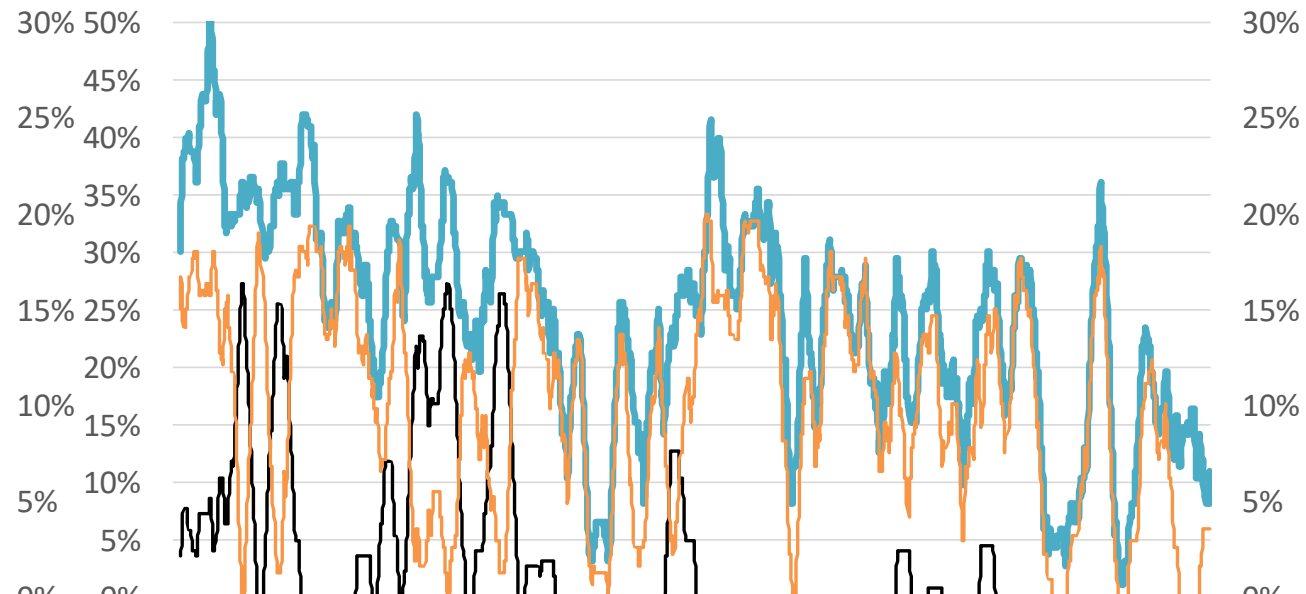

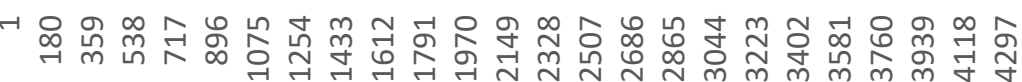


SU Negative 1

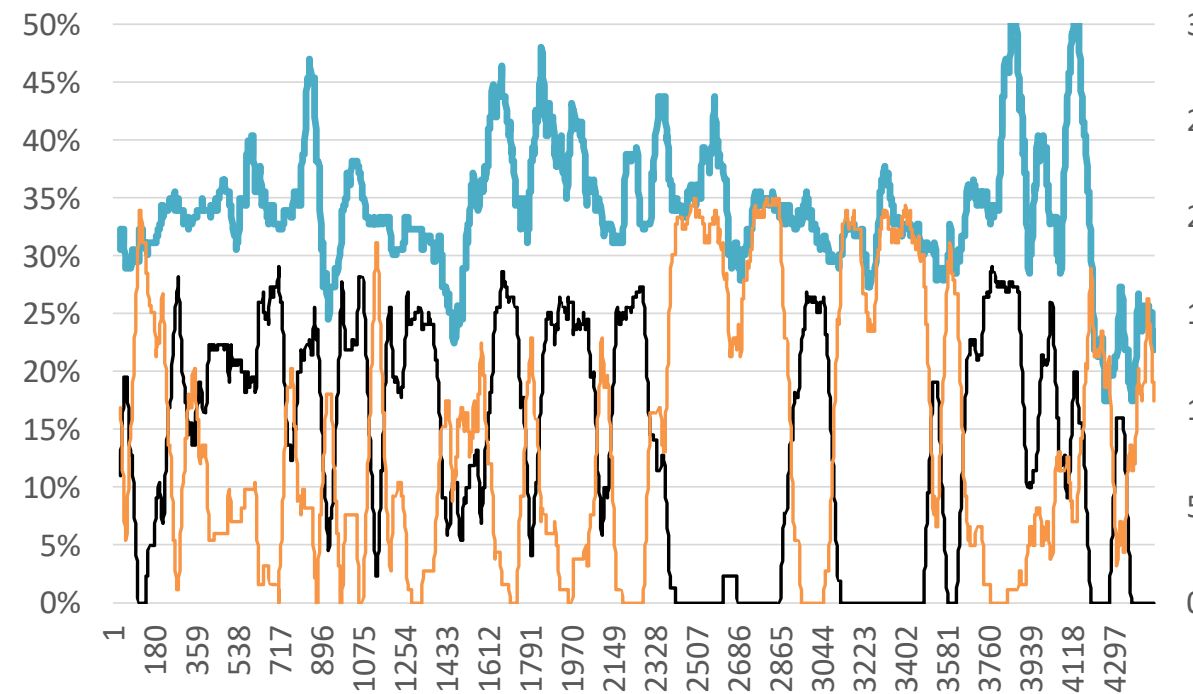

SU Negative 3

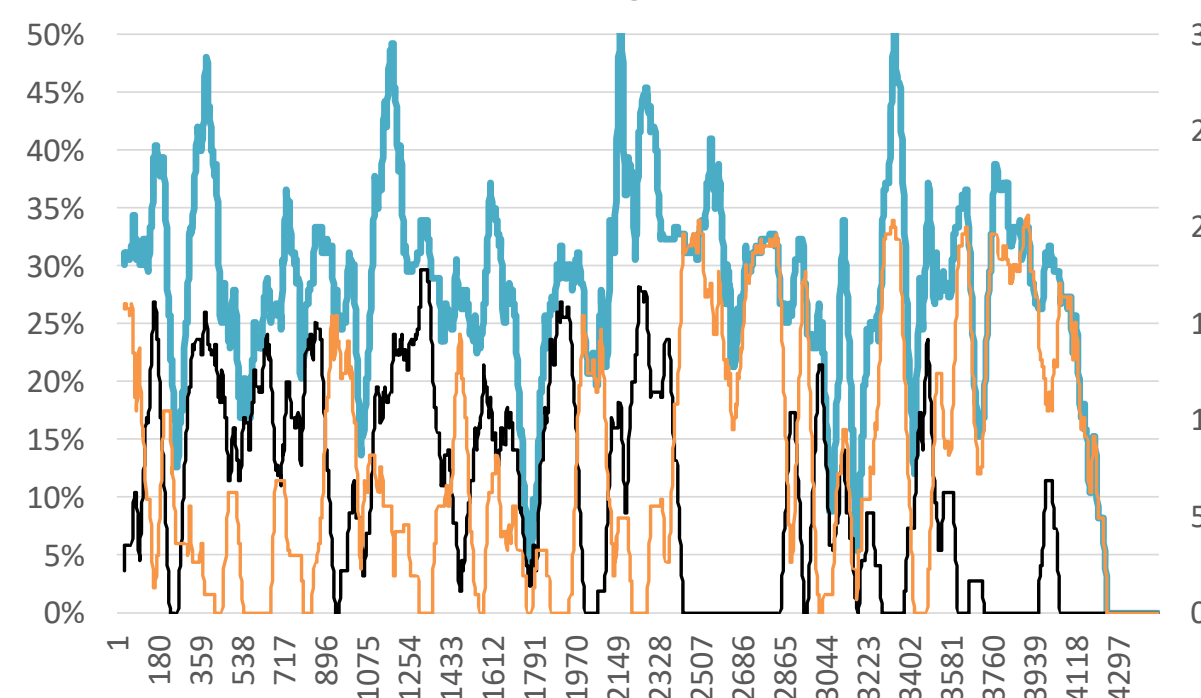

SU Negative 2

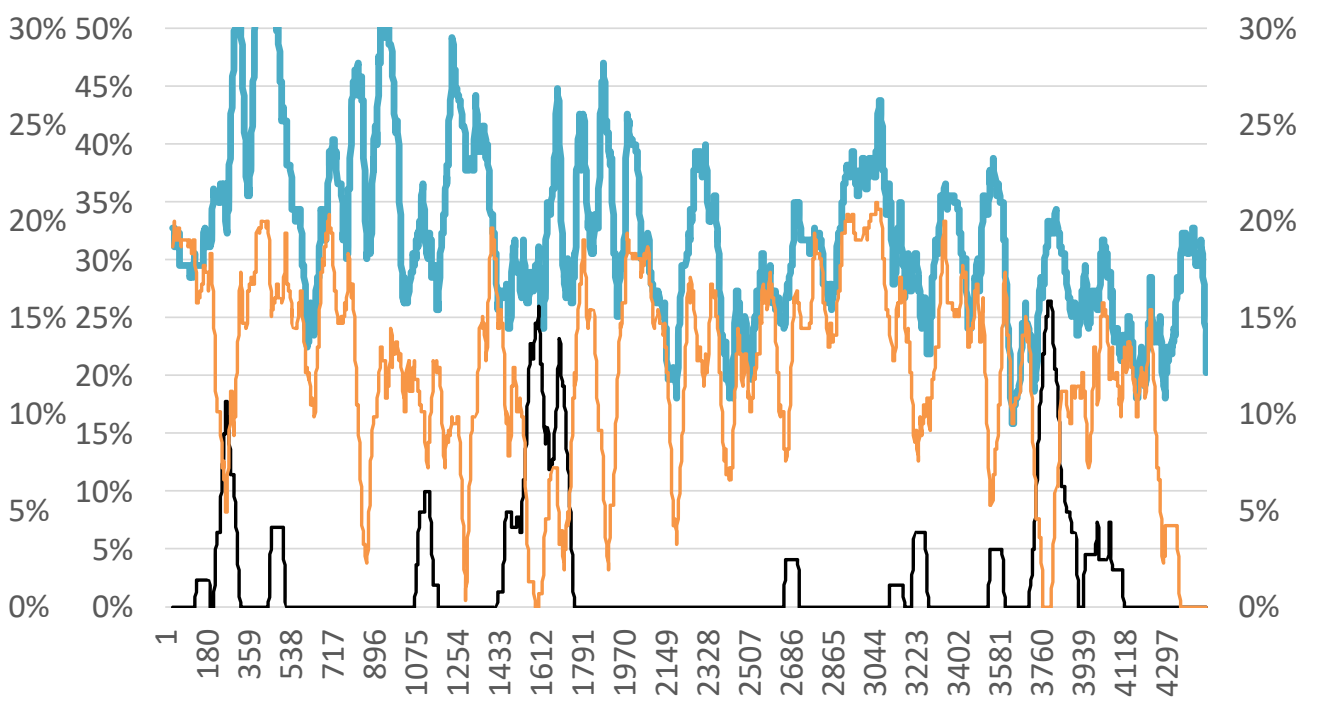

SU Negative 4

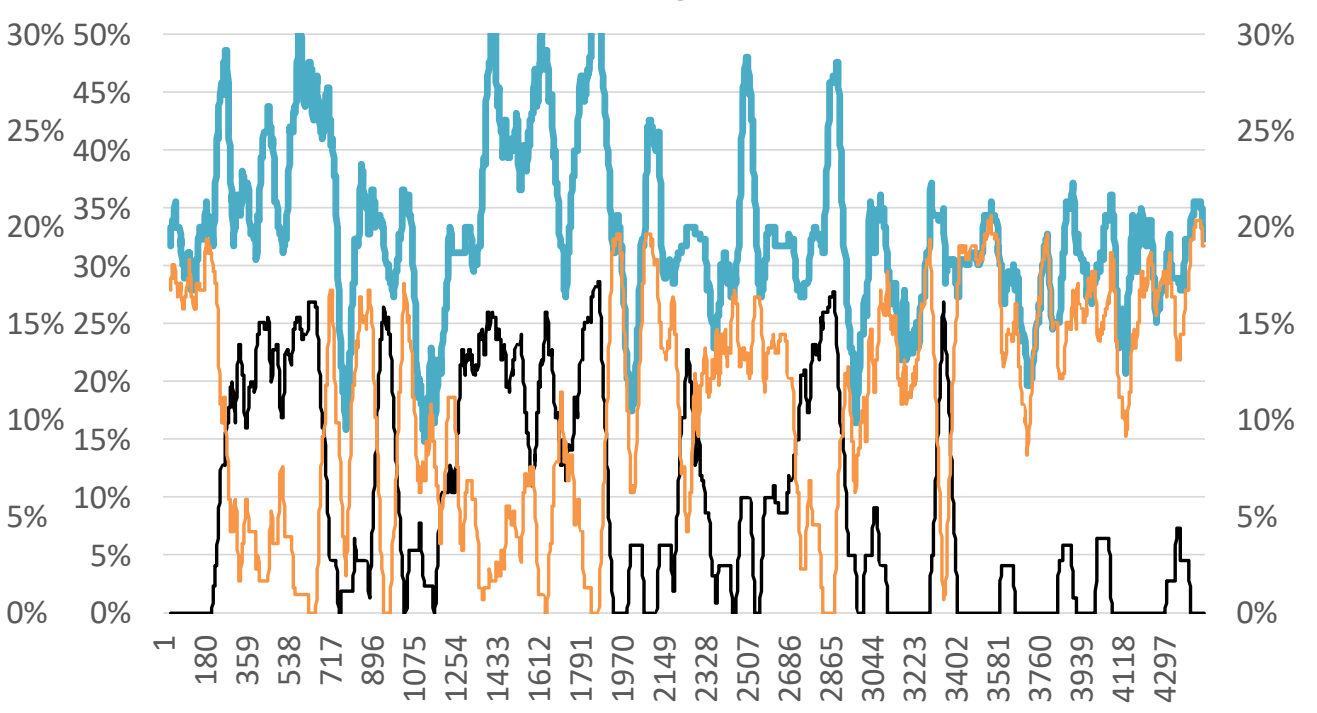

